\title{
Pure Nash Equilibria in Resource Graph Games
}

Tobias Harks

Augsburg University, Universitätsstraße 14

86159 Augsburg, Germany

Max Klimm

Technische Universität Berlin, Straße des 17. Juni 136

10623 Berlin, Germany

Jannik Matuschke

KU Leuven, Research Center for Operations Management

Naamsestraat 69, 3000 Leuven, Belgium
TOBIAS.HARKS@MATH.UNI-AUGSBURG.DE

KLIMM@TU-BERLIN.DE

JANNIK.MATUSCHKE@KULEUVEN.BE

\begin{abstract}
This paper studies the existence of pure Nash equilibria in resource graph games, a general class of strategic games succinctly representing the players' private costs. These games are defined relative to a finite set of resources and the strategy set of each player corresponds to a set of subsets of resources. The cost of a resource is an arbitrary function of the load vector of a certain subset of resources. As our main result, we give complete characterizations of the cost functions guaranteeing the existence of pure Nash equilibria for weighted and unweighted players, respectively.

For unweighted players, pure Nash equilibria are guaranteed to exist for any choice of the players' strategy space if and only if the cost of each resource is an arbitrary function of the load of the resource itself and linear in the load of all other resources where the linear coefficients of mutual influence of different resources are symmetric. This implies in particular that for any other cost structure there is a resource graph game that does not have a pure Nash equilibrium.

For weighted games where players have intrinsic weights and the cost of each resource depends on the aggregated weight of its users, pure Nash equilibria are guaranteed to exist if and only if the cost of a resource is linear in all resource loads, and the linear factors of mutual influence are symmetric, or there is no interaction among resources and the cost is an exponential function of the local resource load.

We further discuss the computational complexity of pure Nash equilibria in resource graph games showing that for unweighted games where pure Nash equilibria are guaranteed to exist, it is coNP-complete to decide for a given strategy profile whether it is a pure Nash equilibrium. For general resource graph games, we prove that the decision whether a pure Nash equilibrium exists is $\Sigma_{2}^{\mathrm{p}}$-complete.
\end{abstract}

\section{Introduction}

Multi-agent systems are characterized by the intricate interplay of the different and sometimes conflicting self-interests of a large number of independent individuals. In order to study the effects of selfish behavior on the overall state of these systems game-theoretic solution concepts are used, most notably the concept of Nash equilibrium. Important questions for the analysis of multi-agents systems are, thus, under which conditions Nash equilibria exist and how they can be computed. For systems with a large number of players (as they 
frequently appear in multi-agent systems modelling economic, traffic, or telecommunication applications), the representation of the games becomes an important issue. For illustration, consider a system with $n$ agents, each with $m$ strategies. Encoding the payoffs of each agent in each of the $m^{n}$ strategy profiles requires the encoding of $n m^{n}$ rational numbers which is impractical even for modest sizes of $n$ and $m$. Fortunately, for many multi-agents systems that arise from practical applications, the agents' payoffs have additional structure that allows for a succinct representation of the payoffs. Examples include extensive form games (Kuhn, 1953), congestion games (Rosenthal, 1973), graphical games (Kearns, Littman, \& Singh, 2001; Ortiz, 2020), games in graphical normal form (Gottlob, Greco, \& Scarcello, 2005), action graph games (Jiang, Leyton-Brown, \& Bhat, 2011), and local effect games (Leyton-Brown \& Tennenholtz, 2003). These representations also often allow for a faster computation of mixed Nash equilibria (Blum, Shelton, \& Koller, 2006).

A general class of games that includes several of the specific classes of games above is the class of resource graph games introduced by Jiang, Chan, and Leyton-Brown (2017). In a resource graph game, we are given a finite set $N=\{1, \ldots, n\}$ of players and a finite set $R=\{1, \ldots, m\}$ of resources. The strategy set available to player $i$ is a set $X_{i} \subseteq\{0,1\}^{m}$ with a succinct representation. ${ }^{1}$ Given a strategy profile $x=\left(\mathbf{x}_{1}, \ldots, \mathbf{x}_{n}\right)$ with $\mathbf{x}_{i} \in X_{i}$ for $i \in N$, let $\mathbf{x}=\sum_{i \in N} \mathbf{x}_{i} \in \mathbb{R}_{\geq 0}^{m}$ denote the configuration profile representing the total number of players using each resource in strategy profile $x$. Then, the private cost of player $i$ is defined as

$$
\pi_{i}(x)=\mathbf{x}_{i}^{\top} \mathbf{c}(\mathbf{x})=\sum_{r \in R} x_{i, r} c_{r}(\mathbf{x}) \quad \text { for all } i \in N,
$$

where $\mathbf{c}: \mathbb{R}_{\geq 0}^{m} \rightarrow \mathbb{R}^{m}$ is an arbitrary function and $x_{i, r}$ indicates whether resource $r$ occurs in player $i$ 's strategy. For most applications, the function $\mathbf{c}: \mathbb{R}_{\geq 0}^{m} \rightarrow \mathbb{R}^{m}$ itself has a succinct representation of the following form. For each resource $r$, let $\bar{B}_{r} \subseteq R$ be an arbitrary subset of resources (possibly also containing $r$ ). Interpreting $B_{r}$ as the local neighborhood of $r$, assume that the function $\mathbf{c}: \mathbb{R}_{\geq 0}^{m} \rightarrow \mathbb{R}^{m}, \mathbf{x} \mapsto\left(c_{1}(\mathbf{x}), \ldots, c_{m}(\mathbf{x})\right)$ has the property that for every resource $r \in R$ the cost $c_{r}$ depends only on the configuration profile of the resources in $B_{r}$, i.e., $c_{r}(\mathbf{x})=c_{r}(\mathbf{y})$ for all $\mathbf{x}, \mathbf{y} \in \mathbb{R}_{\geq 0}^{m}$ with $x_{s}=y_{s}$ for all $s \in B_{r}$. If $\left|B_{r}\right| \leq k$ for all $r \in R$, then the function $\mathbf{c}$ can be encoded by $m n^{k}$ numbers since it suffices to specify for each resource $r$ the value of $c_{r}(\mathbf{x})$ as a function of the $n^{k}$ possible load vectors of the resources in $B_{r}$. A graphical illustration of such a game is obtained by the graph that has the vertex set $R$ and a directed edge from $s$ to $r$ if and only if $s \in B_{r}$, hence, the name resource graph games.

For illustration, consider the following example.

Example 1 (Unweighted resource graph game). There are two players $N=\{1,2\}$ and eight resources $r_{1}, r_{2}, \ldots, r_{8}$. Player 1 has the strategy set $X_{1}=\left\{\mathbf{x}_{1}, \mathbf{x}_{1}^{\prime}\right\}$ with $\mathbf{x}_{1}=\mathbf{1}_{\left\{r_{1}, r_{2}, r_{5}\right\}}$ and $\mathbf{x}_{1}^{\prime}=\mathbf{1}_{\left\{r_{3}, r_{4}, r_{8}\right\}}$ where for a subset $S \subseteq R, \mathbf{1}_{S}$ denotes the indicator vector of set $S$ in $\mathbb{R}^{R}$. The strategy set of player 2 is $X_{2}=\left\{\mathbf{x}_{2}, \mathbf{x}_{2}^{\prime}\right\}$ with $\mathbf{x}_{2}=\mathbf{1}_{\left\{r_{2}, r_{4}, r_{6}\right\}}$ and $\mathbf{x}_{2}^{\prime}=$ $\mathbf{1}_{\left\{r_{1}, r_{3}, r_{7}\right\}}$; see Figure 1a for an illustration. We assume that the cost function of every resource $r$ is of the form $c_{r}(\mathbf{x})=x_{r}+\sum_{s \in B_{r}} x_{s}=2 x_{r}+\sum_{s \in B_{r} \backslash\{r\}} x_{s}$, and that for every

1. Jiang et al. consider a polytopal representation, but also other representations, e.g., in terms of combinatorial structures are possible. 


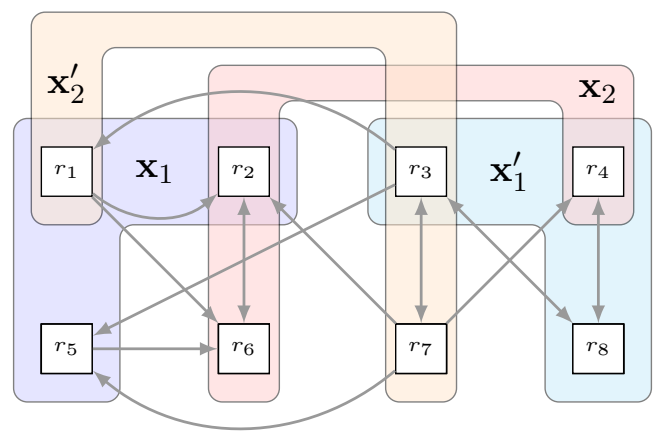

(a) Graphical representation

\begin{tabular}{l|c|c|}
\multicolumn{1}{c}{} & \multicolumn{1}{c}{$\mathbf{x}_{2}$} & $\mathbf{x}_{2}^{\prime}$ \\
\cline { 2 - 3 } $\mathbf{x}_{1}$ & 10,14 & 14,11 \\
\cline { 2 - 3 } $\mathbf{x}_{1}^{\prime}$ & 13,11 & 15,14 \\
\cline { 2 - 3 } & &
\end{tabular}

(b) Bimatrix representation of the unweighted game

\begin{tabular}{l|c|c|} 
& \multicolumn{1}{c}{$\mathbf{x}_{2}$} & \multicolumn{1}{c}{$\mathbf{x}_{2}^{\prime}$} \\
\cline { 2 - 3 } $\mathbf{x}_{1}$ & 13,44 & 21,40 \\
\cline { 2 - 3 } $\mathbf{x}_{1}^{\prime}$ & 16,38 & 20,46 \\
\cline { 2 - 3 } & &
\end{tabular}

(c) Bimatrix representation of the weighted game

Figure 1: Graphical and bimatrix representations of the resource graph game of Example 1.

resource $r \in R$, the neighborhood $B_{r}$ contains the resource $r$ itself and all resources $s \in R$ for which an arc $(s, r)$ is shown in Figure 1a. It is straightforward to verify that we obtain the bimatrix game with private costs shown in Figure 1b. For illustration, we compute the private costs for strategy profile $\left(\mathbf{x}_{1}, \mathbf{x}_{2}\right)$. This strategy profile yields the configuration profile $\mathbf{x}=\mathbf{x}_{1}+\mathbf{x}_{2}=(1,2,0,1,1,1,0,0)^{\top}$. The corresponding cost vector is

$$
\begin{aligned}
\mathbf{c}\left(\mathbf{x}_{1}+\mathbf{x}_{2}\right)= & \left(2 x_{r_{1}}+x_{r_{3}}, 2 x_{r_{2}}+x_{r_{1}}+x_{r_{6}}+x_{r_{7}}, 2 x_{r_{3}}+x_{r_{7}}+x_{r_{8}}, 2 x_{r_{4}}+x_{r_{7}}+x_{r_{8}},\right. \\
& \left.2 x_{r_{5}}+x_{r_{3}}+x_{r_{7}}, 2 x_{r_{6}}+x_{r_{1}}+x_{r_{2}}+x_{r_{5}}, 2 x_{r_{7}}+x_{r_{3}}, 2 x_{r_{8}}+x_{r_{3}}+x_{r_{4}}\right) \\
= & (2,6,0,2,2,6,0,1)^{\top} .
\end{aligned}
$$

The resulting private costs are

$$
\begin{aligned}
& \pi_{1}\left(\mathbf{x}_{1}, \mathbf{x}_{2}\right)=\mathbf{x}_{1}^{\top} \mathbf{c}\left(\mathbf{x}_{1}+\mathbf{x}_{2}\right)=(1,1,0,0,1,0,0,0)^{\top}(2,6,0,2,2,6,0,1)=10 \\
& \pi_{2}\left(\mathbf{x}_{1}, \mathbf{x}_{2}\right)=\mathbf{x}_{2}^{\top} \mathbf{c}\left(\mathbf{x}_{1}+\mathbf{x}_{2}\right)=(0,1,0,1,0,1,0,0)^{\top}(2,6,0,2,2,6,0,1)=14 .
\end{aligned}
$$

From the bimatrix representation in Figure $1 b$ it is easy to see that $\left(\mathbf{x}_{1}, \mathbf{x}_{2}^{\prime}\right)$ is a pure Nash equilibrium of the game.

We also consider a generalization of resource graph games where each player $i$ has an intrinsic weight $w_{i} \in \mathbb{R}_{>0}$ and the strategy of player $i$ is of the form $X_{i}=\left\{w_{i} \mathbf{x}_{i}: \mathbf{x}_{i} \in Y_{i}\right\}$ where $Y_{i} \subseteq\{0,1\}^{m}$ is arbitrary. ${ }^{2}$ For illustration, consider the following example.

Example 2 (Weighted resource graph game). Reconsider the unweighted resource graph game from Example 1 except that the players' weights are $w_{1}=1$ and $w_{2}=2$, respectively,

2. For this generalization, the bound on the encoding of the cost function for the unweighted case does no longer apply in general. However, when player weights are integer and $\left|B_{r}\right| \leq k$ for each of the $m$ resources, the cost function can be encoded with $m w^{k}$ numbers, where $w=\sum_{i \in N} w_{i}$. 
i.e., $X_{1}=\left\{\mathbf{x}_{1}, \mathbf{x}_{1}^{\prime}\right\}$ with $\mathbf{x}_{1}=\mathbf{1}_{\left\{r_{1}, r_{2}, r_{5}\right\}}$ and $\mathbf{x}_{1}^{\prime}=\mathbf{1}_{\left\{r_{3}, r_{4}, r_{8}\right\}}$ and $X_{2}=\left\{\mathbf{x}_{2}, \mathbf{x}_{2}^{\prime}\right\}$ with $\mathbf{x}_{2}=2 \cdot \mathbf{1}_{\left\{r_{2}, r_{4}, r_{6}\right\}}$ and $\mathbf{x}_{2}^{\prime}=2 \cdot \mathbf{1}_{\left\{r_{1}, r_{3}, r_{7}\right\}}$. It is straightforward to verify that we obtain the bimatrix game with private costs shown in Figure 1c. For illustration, we compute the private costs for strategy profile $\left(\mathbf{x}_{1}, \mathbf{x}_{2}\right)$. This strategy profile yields the configuration profile $\mathbf{x}=\mathbf{x}_{1}+\mathbf{x}_{2}=(1,3,0,2,1,2,0,0)^{\top}$. The corresponding cost vector is computed as in $(1)$ and is equal to

$$
\mathbf{c}\left(\mathbf{x}_{1}+\mathbf{x}_{2}\right)=(2,9,0,4,2,9,0,2)^{\top} .
$$

The resulting private costs are

$$
\begin{aligned}
& \pi_{1}\left(\mathbf{x}_{1}, \mathbf{x}_{2}\right)=\mathbf{x}_{1}^{\top} \mathbf{c}\left(\mathbf{x}_{1}+\mathbf{x}_{2}\right)=(1,1,0,0,1,0,0,0)^{\top}(2,9,0,4,2,9,0,2)=13, \\
& \pi_{2}\left(\mathbf{x}_{1}, \mathbf{x}_{2}\right)=\mathbf{x}_{2}^{\top} \mathbf{c}\left(\mathbf{x}_{1}+\mathbf{x}_{2}\right)=(0,2,0,2,0,2,0,0)^{\top}(2,9,0,4,2,9,0,2)=44 .
\end{aligned}
$$

From the bimatrix representation in Figure $1 c$ it is easy to see that the game does not have a pure Nash equilibrium.

Below, we illustrate classes of games that are special cases of resource graph games. We start with the class of unweighted congestion games introduced by Rosenthal (1973) as a model for road traffic and for production with demand-dependent costs. The generalization of unweighted congestion games to weighted players is proposed by Milchtaich (1996).

Example 3 (Congestion games). When the neighborhood of each resource $r$ contains only $r$, i.e., $B_{r}=\{r\}$ for all $r \in R$, the cost of each resource depends only on the number of players using it. We then obtain the class of unweighted congestion games as a special case of unweighted resource graph games and the class of weighted congestion games as a special case of weighted resource graph games.

Another example of a subclass of resource graph games is the class of local effect games as introduced by Leyton-Brown and Tennenholtz (2003). Compared to unweighted congestion games, they are less general in terms of the players' strategies since only singleton strategies are allowed; in terms of the cost structure on the resources, they are more general since the cost of a resource may also depend on the load of other resources. We call a resource graph game a singleton game, if $\left|\left\{r \in R: x_{i, r}>0\right\}\right|=1$ for all $\mathbf{x}_{i}=\left(x_{i, r}\right)_{r \in R} \in X_{i}$ and all $i \in N$.

Example 4 (Local effect games). Local effect games are singleton unweighted resource graph games, where for every resource $r$, there is a function $f_{r}: \mathbb{R}_{\geq 0} \rightarrow \mathbb{R}$ and for every pair of resources $r, s \in R$ such that $s \in B_{r}$, there is a function $f_{r, s}: \mathbb{R}_{\geq 0} \rightarrow \mathbb{R}$ such that $c_{r}(\mathbf{x})=f_{r}\left(x_{r}\right)+\sum_{s \in B_{r}} f_{s, r}\left(x_{s}\right)$.

The following class of action graph games is a generalization of local effect games. They are introduced by Jiang et al. (2011) and generalize local effect games as they allow an arbitrary functional dependence of the cost of a resource on the load of all other resources, yet, they are a subclass of resource graph games. Jiang et al. discuss applications in modelling location games, congestion games, and anonymous games, but the class of games is universal as any strategic game can be represented as an action graph game. Thompson and Leyton-Brown (2017) show how to use action graph games to compute equilibria in position auctions. 
Example 5 (Action graph games). The class of action graph games is equivalent to the class of singleton unweighted resource graph games, i.e., it contains all unweighted resource graph games in which $\left|\left\{r \in R: x_{i, r}>0\right\}\right|=1$ for all $\mathbf{x}_{i}=\left(x_{i, r}\right)_{r \in R} \in X_{i}$ and all $i \in N$.

Finally, we mention that the interdependence of costs of resources on the loads of other resources has a long history in non-atomic traffic models. Dafermos (1971) proposes the use of such cost structures to model the dependencies of the travel times on opposing directions of a two-lane road and on road segments leading to a common crossing. We obtain the following class of congestion games with non-separable costs as the natural atomic counterpart of the non-atomic traffic models with non-separable costs considered in the traffic literature (Dafermos, 1971, 1972; Smith, 1979).

Example 6 (Unweighted network congestion games with non-separable costs). These games are resource graph games, where the set of resources $R$ corresponds to the set of edges of a road network. For every player $i$, the strategy set $X_{i}$ corresponds to the (indicator vectors) of the edge set of all paths between a source node $s^{i}$ and a target node $t^{i}$ in the road network.

In the examples discussed above, a mixed Nash equilibrium is guaranteed to exist due to Nash's theorem (Nash, 1950). However, as discussed, e.g., by Jiang and LeytonBrown (2007), pure Nash equilibria are more favorable as a solution concept as they are easier to implement in practice. For the subclass of local effect games, there is a complete characterization of the cost structures that admit a potential function in all games by Leyton-Brown and Tennenholtz (2003). The existence of a potential function is sufficient for the existence of a pure Nash equilibrium (Monderer \& Shapley, 1996b), but not necessary. In this paper, we are interested in identifying maximal conditions on the cost functions that guarantee the existence of pure Nash equilibria in the larger class of resource graph games.

\subsection{Our Results}

We study the existence of pure Nash equilibria for resource graph games with respect to the non-separable cost structures. This structure inherent to resource graph games distinguishes them from congestion games, where costs are separable in that the cost at each resource only depends on the load of that resource, and for which the existence of Nash equilibria is well-understood. We call a non-empty set $\mathcal{C}$ of cost functions consistent, if every resource graph game with cost functions from $\mathcal{C}$ admits a pure Nash equilibrium. We only require a natural condition on $\mathcal{C}$, namely that $\mathcal{C}$ is closed under composition. This means that for any two functions $\mathbf{c}_{1}, \mathbf{c}_{2} \in \mathcal{C}$, where $\mathbf{c}_{1}=\mathbf{c}_{2}$ is allowed, acting on disjoint resource sets $R_{1}$ and $R_{2}$ with $\left|R_{1}\right|=m_{1}$ and $\left|R_{2}\right|=m_{2}$, respectively, the cost function $\mathbf{c}_{1} \oplus \mathbf{c}_{2}: \mathbb{R}_{\geq 0}^{m_{1}+m_{2}} \rightarrow \mathbb{R}^{m_{1}+m_{2}}$ defined as $\mathbf{c}_{1} \oplus \mathbf{c}_{2}=\left(\mathbf{c}_{1}, \mathbf{c}_{2}\right)$ also belongs to $\mathcal{C}$. This property naturally arises by composing two disjoint subsets $R_{1}$ and $R_{2}$ so that the cost structure within each set of the disjoint union is given by $\mathbf{c}_{1}$ and $\mathbf{c}_{2}$, and there is no interaction between the loads and costs of resources contained in the two different sets. For an intuitive interpretation of this condition for resource graph games played on graphs, see Example 7.

In Section 3, we study the consistency for unweighted resource graph games. Specifically, we show in Theorem 1 that a composition-closed set $\mathcal{C}$ of cost functions is consistent if and only if for each $\mathbf{c} \in \mathcal{C}, \mathbf{c}: \mathbb{Z}_{\geq 0}^{m} \rightarrow \mathbb{R}^{m}$ with $m \in \mathbb{N}$, there are arbitrary func- 
tions $f_{1}, \ldots, f_{m}: \mathbb{Z}_{\geq 0} \rightarrow \mathbb{R}$ and a symmetric matrix $\mathbf{A} \in \mathbb{R}^{m \times m}$ such that

$$
\mathbf{c}(\mathbf{x})=\left(f_{1}\left(x_{1}\right), \ldots, f_{m}\left(x_{m}\right)\right)^{\top}+\mathbf{A} \mathbf{x}
$$

for all load vectors $\mathbf{x} \in \mathbb{Z}_{\geq 0}^{m}$ (except for the irrelevant cost on empty resources). Our result implies in particular that every resource graph game with this cost structure has a pure Nash equilibrium. This generalizes a result of Leyton-Brown and Tennenholtz (2003) who show that for the special case of local effect games with this cost structure, a pure Nash equilibrium exists. Our characterization also implies that for every other cost function $\tilde{\mathbf{c}}$ that does not adhere to this form, there is an unweighted resource graph game with costs defined by $\tilde{\mathbf{c}}$ that does not have a pure Nash equilibrium. For the proof of this result, we construct several highly symmetric resource graph games that allow to derive functional equations on the set of consistent cost functions that combined leave cost functions of the form above as the only possibility.

In Section 4, we study the consistency for weighted resource graph games, providing a full characterization of the cost functions that are consistent for these games. Specifically, we show in Theorem 2 that a composition-closed $\operatorname{set} \mathcal{C}$ of continuous cost functions is consistent if and only if one of the following two cases holds:

(i) There is a common constant $\phi \in \mathbb{R}$ such that for each $\mathbf{c} \in \mathcal{C}, \mathbf{c}: \mathbb{R}_{\geq 0}^{m} \rightarrow \mathbb{R}^{m}$ with $m \in \mathbb{N}$ there are vectors $\mathbf{a}, \mathbf{b} \in \mathbb{R}^{m}$ such that

$$
\mathbf{c}(\mathbf{x})=\left(a_{1} \exp \left(\phi x_{1}\right)+b_{1}, \ldots, a_{m} \exp \left(\phi x_{m}\right)+b_{m}\right)^{\top}
$$

(ii) For each $\mathbf{c} \in \mathcal{C}, \mathbf{c}: \mathbb{R}_{\geq 0}^{m} \rightarrow \mathbb{R}^{m}$ with $m \in \mathbb{N}$, there is a symmetric matrix $\mathbf{A} \in \mathbb{R}^{m \times m}$ and vector $\mathbf{b} \in \mathbb{R}^{m}$ such that

$$
\mathbf{c}(\mathbf{x})=\mathbf{A x}+\mathbf{b} .
$$

Again, our characterization implies that for any other cost function $\tilde{\mathbf{c}}$ there is a weighted resource graph game with costs defined by $\tilde{\mathbf{c}}$ that does not have a pure Nash equilibrium. For the proof of this result, it is insightful to notice that the class of weighted resource graph games contains both the class of unweighted resource graph games and the class of weighted congestion games as subclasses. Harks and Klimm (2012) show that weighted congestion games are guaranteed to have a pure Nash equilibrium if and only if either all cost functions are affine or all cost functions are exponential as in (3). This allows to essentially restrict the search space for cost functions that are consistent for resource graph games to those functions that are either exponential or affine after removing all interdependencies of the costs of resources on the loads of other resources. Combining this insight with our characterization for unweighted resource graph games, then allows to establish the characterization for weighted resource graph games.

In Section 5, we discuss the computational complexity of pure Nash equilibria in resource graph games. We first show that for unweighted resource graph games that are guaranteed to have a pure Nash equilibrium as they satisfy (2), it is coNP-complete to decide for a given strategy profile whether it is a pure Nash equilibrium. This holds even for single player games where the strategy set of the player corresponds to the set of $s$-t-paths in a graph where the resources are associated with the edges, and where $\left|B_{r}\right| \leq 1$ for all $r \in R$. 
The reduction actually only uses affine cost functions as in (4) so that this hardness trivially carries over to unweighted and weighted resource graph games with these costs. For general unweighted resource graph games, we show that the problem to decide whether a given resource graph game has a pure Nash equilibrium is $\Sigma_{2}^{\mathrm{p}}$-complete. This hardness result holds for two-player games where the strategy set of each player $i$ corresponds to the set of $s^{i}$ - $t^{i}$-paths in a graph, $\left|B_{r}\right| \leq 1$ for all $r \in R$, and all cost functions are linear. It is interesting to contrast this hardness result with a result of Gottlob et al. (2005), who show that it is $\Sigma_{2}^{\mathrm{p}}$-complete whether a strategic game has a strong Nash equilibrium. While their result concerns stability against coordinated deviations of groups of players with polynomially large strategy sets, our hardness result concerns stability against single-player deviations for a player with an exponentially large strategy set.

\subsection{Significance}

When designing multi-agent systems, stability is a major concern. As a consequence, system designers are interested in implementing systems that have at least one pure Nash equilibrium. However, our $\Sigma_{2}^{\mathrm{p}}$-completeness result for the problem to decide whether a pure Nash equilibrium exists, makes it unlikely that this problem can be solved in reasonable time for large instances, even with techniques tailored to solve NP-complete problems such as SAT-solving or integer programming. This issue provides additional justification to stick with cost structures that guarantee the existence of pure Nash equilibria which we completely characterize for both unweighted and weighted players. As our proofs reveal, in addition to guaranteeing the existence of pure Nash equilibria, these cost functions actually yield the existence of a weighted potential function; see Monderer and Shapley (1996b) for a definition. Weighted potential games have additional desirable properties, e.g., myopic improvement steps or fictitious play converge to an equilibrium (Monderer \& Shapley, 1996a).

\subsection{Related Work}

As discussed in Example 3, unweighted congestion games are a subclass of resource graph games. Rosenthal (1973) shows that every unweighted congestion game with separable costs has a pure Nash equilibrium. Milchtaich (1996) proposes two generalizations of unweighted congestion games. In the first generalization, called weighted congestion games, each player has a weight and the cost of each resource depends on the aggregated weight of its users. In the second generalization, called congestion games with player-specific costs, every player has an individual cost function for each resource. Both generalizations alone still admit a pure Nash equilibrium for singleton congestion games, but the combination of both may fail to have a pure Nash equilibrium, even for singleton games. The positive result for singletons is generalized by Ackermann, Röglin, and Vöcking (2009) to games where the strategy set of each player corresponds to the set of bases of a matroid. Weighted congestion games with general strategy spaces may fail to have a pure Nash equilibrium (Goemans, Mirrokni, \& Vetta, 2005; Libman \& Orda, 2001), but have a pure Nash equilibrium for affine costs or exponential costs (Harks \& Klimm, 2012; Harks, Klimm, \& Möhring, 2011; Fotakis, Kontogiannis, \& Spirakis, 2005; Panagopoulou \& Spirakis, 2006). Local effect games are introduced by Leyton-Brown and Tennenholtz (2003) who show that a pure Nash 
equilibrium exists when the mutual influence of different resources on the cost is linear and symmetric. Dunkel and Schulz (2008) show that for these games the computation of a pure Nash equilibrium is PLS-complete. They also show that for both local effect games with non-linear mutual effects and weighted congestion games with arbitrary cost functions, it is NP-hard to decide whether a pure Nash equilibrium exists. The PLS-completeness of computing a pure Nash equilibrium in unweighted congestion games with affine costs due to Ackermann, Röglin, and Vöcking (2008) carries over to the weighted case.

The class of action graph games is introduced by Bhat and Leyton-Brown (2004) and Jiang et al. (2011) as a generalization of local-effect games. They show that every strategic game can be represented as an action graph game. Daskalakis, Schoenebeck, Valiant, and Valiant (2009) give a fully polynomial-time approximation scheme (FPTAS) for computing an approximate mixed equilibrium in action graph games with constant degree, constant treewidth, and a constant number of agent types. They also give several hardness results for games that violate one of these requirements. Jiang and Leyton-Brown (2007) show that for symmetric action graph games played on a graph of bounded treewidth, it can be decided efficiently whether a pure Nash equilibrium exists while the problem is NP-hard to decide in general.

Resource graph games are introduced by Jiang et al. (2017) as a further generalization of action graph games. Chan and Jiang (2018) give an FPTAS for computing an approximate Nash equilibrium in resource graph games with a constant number of player types and further restrictions on the strategy sets.

Congestion games with non-atomic players where the load of one resource has an impact on the cost of another resource are usually called congestion games with non-separable costs. They are first proposed by Dafermos $(1971,1972)$. She shows that the equilibrium condition can be formulated as an optimization problem, if the Jacobian of the cost function is symmetric. This condition is reminiscent of one the conditions that we identify as a necessary condition for the existence of a pure Nash equilibrium in Lemma 3. Smith (1979) provides a variational inequality for the non-symmetric case. Perakis (2007) studies the price of anarchy of non-atomic congestion games with linear non-separable costs of the form $\mathbf{c}(\mathbf{x})=\mathbf{A x}+\mathbf{b}$.

\section{Preliminaries}

For an integer $k \in \mathbb{N}$, let $[k]:=\{1, \ldots, k\}$. Let $N=[n]$ be a finite set of $n$ players and $R=[m]$ be a finite set of $m$ resources. For each player $i$, the set of strategies available to player $i$ is an arbitrary set $X_{i} \subseteq\{0,1\}^{m}$. We call $x=\left(\mathbf{x}_{1}, \ldots, \mathbf{x}_{n}\right)$ with $\mathbf{x}_{i} \in X_{i}$ for all $i \in N$ a strategy profile and $X=X_{1} \times \cdots \times X_{n}$ the strategy space. We use standard game theory notation; for a strategy profile $x \in X$, we write $x=\left(\mathbf{x}_{i}, x_{-i}\right)$ meaning that $\mathbf{x}_{i}$ is the strategy that player $i$ plays in $x$ and $x_{-i}$ is the partial strategy profile of all players except $i$. Every strategy profile $x=\left(\mathbf{x}_{1}, \ldots, \mathbf{x}_{n}\right) \in X$ induces a load vector $\mathbf{x}=\sum_{i \in[n]} \mathbf{x}_{i} \in \mathbb{R}_{\geq 0}^{m}$. For a set $S \subseteq R=[m]$, we denote by $\mathbf{1}_{S}$ the indicator vector of set $S$ in $\mathbb{R}^{m}$. We are further given a cost function $\mathbf{c}: \mathbb{R}_{\geq 0}^{m} \rightarrow \mathbb{R}^{m}$. Intuitively, the function $\mathbf{c}$ maps a load vector $\mathbf{x} \in \mathbb{R}_{\geq 0}^{m}$ to a cost vector $\mathbf{c}(\mathbf{x}) \in \mathbb{R}^{m}$, i.e., $\mathbf{c}(\mathbf{x})=\left(c_{1}(\mathbf{x}), \ldots, c_{m}(\mathbf{x})\right)^{\top}$ and for a resource $r \in R$ the cost experienced by players using $r$ when the congestion vector is $\mathbf{x}$ is $c_{r}(\mathbf{x})$. A resource graph game is a strategic game $G=\left(N, X,\left(\pi_{i}\right)_{i \in N}\right)$ where the private cost of player $i$ in 
strategy profile $x \in X$ is defined as

$$
\pi_{i}(x)=\mathbf{x}_{i}^{\top} \mathbf{c}(\mathbf{x})=\sum_{r \in R} x_{i, r} c_{r}(\mathbf{x})
$$

with $x_{i, r}$ denoting the $r$ th component of $\mathbf{x}_{i}$.

We also consider a generalization of resource graph games to weighted players. In a weighted resource graph game, every player $i \in N$ has a weight $w_{i} \in \mathbb{R}_{>0}$. The strategy set of player $i$ is then defined as $X_{i}=\left\{w_{i} \mathbf{y}_{i}: \mathbf{y}_{i} \in Y_{i}\right\}$ where $Y_{i} \subseteq\{0,1\}^{m}$ is arbitrary. Compared to unweighted resource graph games, in a weighted resource graph game, the set of vectors in the strategy of player $i$ is multiplied with the scalar $w_{i}$. The weighted resource graph game is then the strategic game $G=\left(N, X,\left(\pi_{i}\right)_{i \in N}\right)$ where $\pi_{i}(x)=\mathbf{x}_{i}^{\top} \mathbf{c}(\mathbf{x})$ for $x \in X$ with $\mathbf{x}=\sum_{i \in[n]} \mathbf{x}_{i}$ as before (note that each $\mathbf{x}_{i} \in X_{i}$ has entries in $\left\{0, w_{i}\right\}$ and thus the load vector $\mathbf{x}$ specifies the total weight on each resource).

A strategy profile $x \in X$ is a pure Nash equilibrium, if $\pi_{i}(x) \leq \pi_{i}\left(\mathbf{y}_{i}, x_{-i}\right)$ for all $i \in N$ and $\mathbf{y}_{i} \in X_{i}$. For a non-empty set of cost functions $\mathcal{C}$, we are interested in establishing conditions on $\mathcal{C}$ that ensure that every resource graph game with cost functions from $\mathcal{C}$ admits pure Nash equilibria.

As a mild technical assumption on $\mathcal{C}$, we will require that the set $\mathcal{C}$ is closed under composition of cost functions. Intuitively, this composition allows to arbitrarily embed several cost functions from $\mathcal{C}$, defined on distinct resource sets, in a common larger resource set, thus composing arbitrary games whose cost structure locally consists of functions from $\mathcal{C}$ as building blocks; see Example 7 below. To make this formal, we say that $\mathcal{C}$ is closed under composition if for any $\mathbf{c}, \mathbf{c}^{\prime} \in \mathcal{C}$ with $\mathbf{c}: \mathbb{R}_{\geq 0}^{m} \rightarrow \mathbb{R}^{m}$ and $\mathbf{c}^{\prime}: \mathbb{R}_{\geq 0}^{m^{\prime}} \rightarrow \mathbb{R}^{m^{\prime}}$, also the function $\mathbf{c} \oplus \mathbf{c}^{\prime}: \mathbb{R}_{\geq 0}^{m+m^{\prime}} \rightarrow \mathbb{R}_{\geq 0}^{m+m^{\prime}}$ defined as $\mathbf{c} \oplus \mathbf{c}^{\prime}(\mathbf{x}, \mathbf{y})=\left(\mathbf{c}(\mathbf{x}), \mathbf{c}^{\prime}(\mathbf{y})\right)$ is also contained in $\mathcal{C}$. This is an intuitive property of a set of functions $\mathcal{C}$ for the following reasons. The cost functions c, $\mathbf{c}^{\prime}$ each define a cost structure on sets of resources $R, R^{\prime}$ with $|R|=m$ and $\left|R^{\prime}\right|=m^{\prime}$. The cost function $\mathbf{c} \oplus \mathbf{c}^{\prime}: \mathbb{R}_{>0}^{m+m^{\prime}} \rightarrow \mathbb{R}^{m+m^{\prime}}$ then defines a cost structure on the disjoint union of $R$ and $R^{\prime}$, where the cost structure within each set of the disjoint union is given by $\mathbf{c}$ or $\mathbf{c}^{\prime}$, and there is no interaction between the loads and costs of resources contained in the two different sets. In particular, for any $k \in \mathbb{N}$ and any $\mathbf{c} \in \mathcal{C}, \mathbf{c}: \mathbb{R}_{>0}^{m} \rightarrow \mathbb{R}^{m}$, the $k$-fold disjoint union $\mathbf{c} \oplus \cdots \oplus \mathbf{c}: \mathbb{R}_{\geq 0}^{k m} \rightarrow \mathbb{R}^{k m}$ is contained in $\mathcal{C}$. In the following, we denote the $k$-fold disjoint union of $\mathbf{c}$ by $\mathbf{c}^{k}$.

For a set $\mathcal{C}$ of cost functions as above, we say that $\mathcal{C}$ is consistent for unweighted resource graph games, if for every $\mathbf{c} \in \mathcal{C}$, we have that every unweighted resource graph game with costs given by c has a pure Nash equilibrium. Recall that when c : $\mathbb{R}_{\geq 0}^{m} \rightarrow \mathbb{R}^{m}$ with $m \in \mathbb{N}$, then every unweighted resource graph game with costs given by $\mathbf{c}$ has $m$ resources. Consistency for weighted resource graph games is defined analogously.

Example 7. To illustrate the concept of composition introduced above, suppose we are interested in modeling road networks as resource graph games. Such a network consists of individual road segments and junctions. For simplicity, assume each road segment consists of a single lane in each direction and each junction is a four-way intersection without left turns. A single road segment then can be modeled by two resources representing the opposing lanes. The travel time on each lane is a function $\mathbf{c}: \mathbb{R}^{2} \rightarrow \mathbb{R}^{2}$ of the load on the lane itself and the opposing lane (e.g., traffic on the opposing lane prevents overtaking) as proposed 


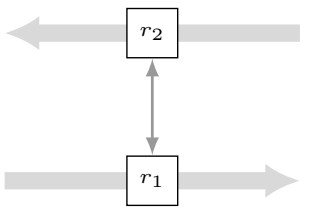

(a) Opposing lanes

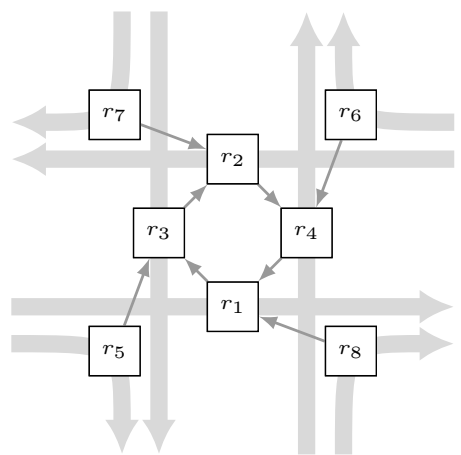

(b) Four-way intersection

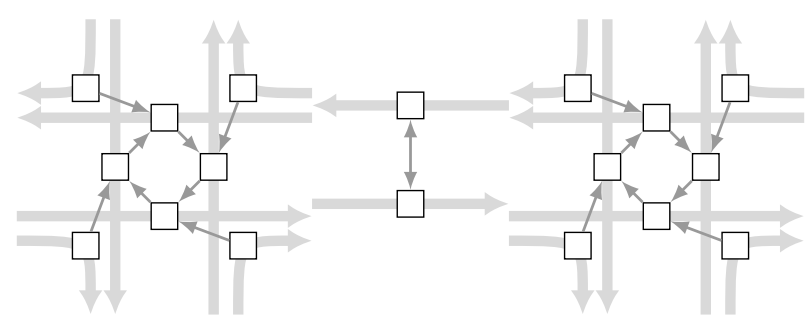

(c) Network composed of opposing lanes and four-way junctions

Figure 2: Illustration of composition of cost functions.

by (Dafermos, 1971); see Figure 2 (a). Similarly, a four-way junction without left turns can be modeled by 8 resources (each representing a possible combination of entry and exit from the junction), with the travel times for passing the junction determined by a function c $: \mathbb{R}^{8} \rightarrow \mathbb{R}^{8}$ modeling turn priority; see Figure $2(b)$. For both the cost functions at road segments and junctions, we may impose assumptions, limiting the respective cost functions to certain classes of 2-and 8-dimensional functions respectively, which we include in the set $\mathcal{C}$. An entire road network, with multiple road segments and junctions can be modeled by composing multiple functions from $\mathcal{C}$ to obtain a resource set of size $2 \ell+8 \ell^{\prime}$, where $\ell$ and $\ell^{\prime}$ is the number of road segments and crossings, respectively; see Figure 2 for an example of such a network with $\ell=1$ and $\ell^{\prime}=2$. Assuming that $\mathcal{C}$ is closed under composition, it thus contains the cost functions of all possible road networks that are built in this way. Determining under which conditions $\mathcal{C}$ is consistent corresponds to finding out conditions on the cost functions for road segments and junctions that guarantee the existence of a pure Nash equilibrium for any resource graph game played on any road network constructed in this way.

\section{Resource Graph Games with Unweighted Players}

In this section, we consider unweighted resource graph games. Since in such a game, the load on each resource is a nonnegative integer, it is without loss of generality to assume that the domain of all cost functions is the non-negative integer lattice, that is, they are of 
the form $\mathbf{c}: \mathbb{Z}_{\geq 0}^{m} \rightarrow \mathbb{R}^{m}$ for some $m \in \mathbb{N}$. Our main result gives a complete characterization of consistency for unweighted resource graph games.

Note that, if a particular resource is not used by any player in a given strategy profile, then no player experiences the cost at that resource for the corresponding load vector. Thus this value is irrelevant for the definition of the game. We therefore say that two cost functions $\mathbf{c}$ and $\mathbf{c}^{\prime}$ are equivalent if $c_{r}(\mathbf{x})=c_{r}^{\prime}(\mathbf{x})$ for all $r \in R$ and all $\mathbf{x} \in \mathbb{R}_{\geq 0}^{m}$ with $x_{r}>0$. Using this notion of equivalence, we can state our characterization.

Theorem 1. Let $\mathcal{C}$ be a set of cost functions that is closed under composition. Then the following two statements are equivalent:

(i) $\mathcal{C}$ is consistent for unweighted resource graph games.

(ii) For each $\mathbf{c} \in \mathcal{C}$ with $\mathbf{c}: \mathbb{Z}_{\geq 0}^{m} \rightarrow \mathbb{R}^{m}$ for some $m \in \mathbb{N}$, there are functions $f_{1}, \ldots, f_{m}$ : $\mathbb{Z}_{\geq 0} \rightarrow \mathbb{R}$ and a symmetric matrix $\mathbf{A} \in \mathbb{R}^{m \times m}$ such that $\mathbf{c}$ is equivalent to the cost function $\mathbf{c}^{\prime}$ defined by

$$
\mathbf{c}^{\prime}(\mathbf{x})=\left(f_{1}\left(x_{1}\right), \ldots, f_{m}\left(x_{m}\right)\right)^{\top}+\mathbf{A} \mathbf{x} .
$$

In particular, the set $\mathcal{C}^{*}$ of all cost functions of the form (6) is (up to equivalence) the unique maximal set of cost functions that is closed under composition and consistent for unweighted resource graph games.

We subdivided the proof of both directions in the following subsections.

\subsection{Proof of Theorem 1: (ii). $\Rightarrow$ (i).}

We first prove that statement $(i i)$. of Theorem 1 implies consistency of $\mathcal{C}$. Observe that any composition of functions of form (6) is again of form (6). It is thus sufficient to show existence of a pure Nash equilibrium for any unweighted resource graph game with a cost function of this form.

Lemma 1. Let $G$ be an unweighted resource graph game on $m$ resources with cost function $\mathbf{c}: \mathbb{R}_{\geq 0}^{m} \rightarrow \mathbb{R}^{m}$ given by $\mathbf{c}(\mathbf{x})=\left(f_{1}\left(x_{1}\right), \ldots, f_{m}\left(x_{m}\right)\right)^{\top}+\mathbf{A x}$, where $f_{1}, \ldots, f_{m}: \mathbb{Z}_{\geq 0} \rightarrow \mathbb{R}$ are arbitrary functions and $\mathbf{A} \in \mathbb{R}^{m \times m}$ is a symmetric matrix. Then $G$ has a pure Nash equilibrium.

Proof. The proof uses an exact potential function that, for the special class of localeffect games, coincides with the potential function given by Leyton-Brown and Tennenholtz (2003). Fix an arbitrary unweighted resource graph game $G$ whose cost is determined by $\mathbf{c} \in \mathcal{C}$ and an arbitrary strategy profile $x \in X$. Consider the process of adding the players to the game in order $1, \ldots, n$ and let us sum their private costs. In the following, we write $\mathbf{x}_{\leq i}=\sum_{j \in N: j \leq i} \mathbf{x}_{j}$ for the load vector of players up to $i$. Let $P(x)$ be the sum of the private costs of the players added to the game when adding them in order $1, \ldots, n$. We define the function $\mathbf{f}: \mathbb{Z}_{\geq 0}^{m} \rightarrow \mathbb{R}^{m}$ as $\mathbf{f}(\mathbf{x})=\left(f_{1}\left(x_{1}\right), \ldots, f_{m}\left(x_{m}\right)\right)^{\top}$ and obtain

$$
P(x)=\sum_{i \in N} \mathbf{x}_{i}^{\top}\left[\mathbf{f}\left(\mathbf{x}_{\leq i}\right)+\mathbf{A} \mathbf{x}_{\leq i}\right] .
$$


We have

$$
\sum_{i \in N} \mathbf{x}_{i}^{\top} \mathbf{f}\left(\mathbf{x}_{\leq i}\right)=\sum_{r \in R} \sum_{k=1}^{x_{r}} f_{r}(k)
$$

as well as

$$
\begin{aligned}
\sum_{i \in N} \mathbf{x}_{i}^{\top} \mathbf{A} \mathbf{x}_{\leq i} & =\sum_{i \in N} \mathbf{x}_{i}^{\top} \mathbf{A}\left(\sum_{j \in N: j \leq i} \mathbf{x}_{j}\right) \\
& =\frac{1}{2} \sum_{i \in N} \sum_{j \in N} \mathbf{x}_{i}^{\top} \mathbf{A} \mathbf{x}_{j}+\frac{1}{2} \sum_{i \in N} \mathbf{x}_{i}^{\top} \mathbf{A} \mathbf{x}_{i} \\
& =\frac{1}{2} \mathbf{x}^{\top} \mathbf{A} \mathbf{x}+\frac{1}{2} \sum_{i \in N} \mathbf{x}_{i}^{\top} \mathbf{A} \mathbf{x}_{i},
\end{aligned}
$$

where for the second equation we used the symmetry of $\mathbf{A}$. We obtain

$$
P(x)=\sum_{r \in R} \sum_{k=1}^{x_{r}} f_{r}(k)+\frac{1}{2} \mathbf{x}^{\top} \mathbf{A} \mathbf{x}+\frac{1}{2} \sum_{i \in N} \mathbf{x}_{i}^{\top} \mathbf{A} \mathbf{x}_{i} .
$$

This shows that $P(x)$ is invariant under a reordering of the players. Next, consider a deviation of an arbitrary player. Since $P(x)$ is invariant under a reordering of the players, it is without loss of generality to assume that player $n$ deviates. We obtain

$$
\begin{aligned}
P\left(\mathbf{y}_{n}, x_{-n}\right)-P(x)= & \mathbf{y}_{n}^{\top}\left[\mathbf{f}\left(\mathbf{x}_{\leq n-1}+\mathbf{y}_{n}\right)+\mathbf{A}\left(\mathbf{x}_{\leq n-1}+\mathbf{y}_{n}\right)\right] \\
& -\mathbf{x}_{n}^{\top}\left[\mathbf{f}\left(\mathbf{x}_{\leq n}\right)+\mathbf{A}\left(\mathbf{x}_{\leq n}\right)\right] \\
= & \pi_{n}\left(\mathbf{y}_{n}, x_{-n}\right)-\pi_{n}(x) .
\end{aligned}
$$

We conclude that $P$ is an exact potential function. Monderer and Shapley (1996b) show that finite exact potential games admit a pure Nash equilibrium. Since $G$ was chosen arbitrarily, the result follows.

\subsection{Proof of Theorem 1: (i). $\Rightarrow$ (ii).}

In the following, we show that statement (ii). of Theorem 1 is a necessary condition for the consistency of $\mathcal{C}$. We prove this by constructing for any given $\mathbf{c} \in \mathcal{C}$ a family of different resource graph games whose cost functions are 4-fold compositions of c. All these games will have the following symmetry property, which we will use to establish that $\mathbf{c}$ is indeed of the form (6).

Definition 1. Let $\alpha, \beta \in \mathbb{R}$. We say a game $G=\left(N, X,\left(\pi_{i}\right)_{i \in N}\right)$ is $(\alpha, \beta)$-symmetric for players $i, j \in N$, if for any strategy profile $x \in X$, the following two statements are fulfilled:

(i) $\left\{\pi_{i}(x), \pi_{j}(x)\right\}=\{\alpha, \beta\}$.

(ii) There are $\mathbf{y}_{i} \in X_{i}$ and $\mathbf{y}_{j} \in X_{j}$ such that $\pi_{i}\left(\mathbf{y}_{i}, x_{-i}\right)=\pi_{j}(x)$ and $\pi_{j}\left(\mathbf{y}_{j}, x_{-j}\right)=\pi_{i}(x)$. 
Note that $\alpha$ and $\beta$ in the above definition do not depend on the strategy profile $x$, but rather that (i) for the same $\alpha$ and $\beta$ must hold for all $x$. The following lemma shows a key property for $(\alpha, \beta)$-symmetric games.

Lemma 2. If a game $G$ is $(\alpha, \beta)$-symmetric for players $i, j \in N$ and admits a pure Nash equilibrium, then $\alpha=\beta$.

Proof. Let $x \in X$ be a pure Nash equilibrium for $G$. Because $G$ is $(\alpha, \beta)$-symmetric for $i$ and $j$, there are are $\mathbf{y}_{i} \in X_{i}$ and $\mathbf{y}_{j} \in X_{j}$ such that $\pi_{i}\left(\mathbf{y}_{i}, x_{-i}\right)=\pi_{j}(x)$ and $\pi_{j}\left(\mathbf{y}_{j}, x_{-j}\right)=\pi_{i}(x)$. Because $x$ is a pure Nash equilibrium, we obtain

$$
\pi_{i}(x) \leq \pi_{i}\left(\mathbf{y}_{i}, x_{-i}\right)=\pi_{j}(x) \leq \pi_{j}\left(\mathbf{y}_{j}, x_{-j}\right)=\pi_{i}(x)
$$

and hence $\pi_{i}(x)=\pi_{j}(x)$. Note that $(\alpha, \beta)$-symmetry of $G$ further implies $\{\alpha, \beta\}=$ $\left\{\pi_{i}(x), \pi_{j}(x)\right\}$ and therefore $\alpha=\beta$.

We proceed to prove a first functional equation that needs to be satisfied for a set of consistent cost functions that is closed under composition. The equation states that a discrete version of the Jacobian of the cost function must be symmetric. For the proof, we construct a suitable $(\alpha, \beta)$-symmetric game.

Lemma 3. Let $\mathcal{C}$ be closed under composition and consistent for unweighted resource graph games. Then, for all $\mathbf{c} \in \mathcal{C}, \mathbf{c}: \mathbb{Z}_{\geq 0}^{m} \rightarrow \mathbb{R}^{m}$ with $m \in \mathbb{N}$, we have

$$
c_{r}\left(\mathbf{x}+\mathbf{1}_{\{r, s\}}\right)-c_{r}\left(\mathbf{x}+\mathbf{1}_{r}\right)=c_{s}\left(\mathbf{x}+\mathbf{1}_{\{r, s\}}\right)-c_{s}\left(\mathbf{x}+\mathbf{1}_{s}\right)
$$

for all $r, s \in[m]$ and all $\mathbf{x} \in \mathbb{Z}_{\geq 0}^{m}$.

Proof. Let $\mathbf{c} \in \mathcal{C}, r, s \in[m]$ with $r \neq s$, let $\mathbf{x} \in \mathbb{Z}_{\geq 0}^{m}$ be arbitrary. Since $\mathcal{C}$ is closed under composition, we have that $\mathbf{c}^{4}: \mathbb{Z}_{\geq 0}^{4 m} \rightarrow \mathbb{R}^{4 m}$ is also contained in $\mathcal{C}$. Consider the following game with $4 m$ resources and cost function $\mathbf{c}^{4}$. For $k \in[4]$ and $t \in[m]$ we denote the $k$-th copy of resource $t$ by $t_{k}$. For each original resource $t \in[m]$, there are $x_{t}$ dummy players whose only strategy is $\sum_{k \in[4]} \mathbf{1}_{t_{k}}$. In addition there are two players 1 and 2 with strategy sets $X_{1}=\left\{\mathbf{1}_{\left\{r_{1}, s_{2}\right\}}, \mathbf{1}_{\left\{r_{3}, s_{4}\right\}}\right\}$ and $X_{2}=\left\{\mathbf{1}_{\left\{r_{2}, s_{3}\right\}}, \mathbf{1}_{\left\{r_{4}, s_{1}\right\}}\right\}$. See Figure 3 for a depiction of the strategy space.

Let $x_{-\{1,2\}}$ be the unique strategy profile of the dummy players. Note that there are only four strategy profiles, these are:

$$
\begin{array}{ll}
x^{(1,1)}=\left(\mathbf{1}_{\left\{r_{1}, s_{2}\right\}}, \mathbf{1}_{\left\{r_{2}, s_{3}\right\}}, x_{-\{1,2\}}\right), & x^{(1,2)}=\left(\mathbf{1}_{\left\{r_{1}, s_{2}\right\}}, \mathbf{1}_{\left\{r_{4}, s_{1}\right\}}, x_{-\{1,2\}}\right), \\
x^{(2,1)}=\left(\mathbf{1}_{\left\{r_{3}, s_{4}\right\}}, \mathbf{1}_{\left\{r_{2}, s_{3}\right\}}, x_{-\{1,2\}}\right), & x^{(2,2)}=\left(\mathbf{1}_{\left\{r_{3}, s_{4}\right\}}, \mathbf{1}_{\left\{r_{4}, s_{1}\right\}}, x_{-\{1,2\}}\right) .
\end{array}
$$

Define $\alpha=c_{r}\left(\mathbf{x}+\mathbf{1}_{\{r, s\}}\right)+c_{s}\left(\mathbf{x}+\mathbf{1}_{s}\right)$ and $\beta=c_{r}\left(\mathbf{x}+\mathbf{1}_{r}\right)+c_{s}\left(\mathbf{x}+\mathbf{1}_{\{r, s\}}\right)$.

Noting that $x_{r_{1}}^{(1,1)}=x_{r}+1, x_{s_{1}}^{(1,1)}=x_{s}, x_{r_{2}}^{(1,1)}=x_{r}+1, x_{s_{2}}^{(1,1)}=x_{s}+1, x_{r_{3}}^{(1,1)}=x_{r}$, and $x_{s_{3}}^{(1,1)}=x_{s}+1$, we obtain

$$
\begin{aligned}
& \pi_{1}\left(x^{(1,1)}\right)=c_{r_{1}}^{4}\left(\mathbf{x}^{(1,1)}\right)+c_{s_{2}}^{4}\left(\mathbf{x}^{(1,1)}\right)=c_{r}\left(\mathbf{x}+\mathbf{1}_{r}\right)+c_{s}\left(\mathbf{x}+\mathbf{1}_{\{r, s\}}\right)=\beta, \\
& \pi_{2}\left(x^{(1,1)}\right)=c_{r_{2}}^{4}\left(\mathbf{x}^{(1,1)}\right)+c_{s_{3}}^{4}\left(\mathbf{x}^{(1,1)}\right)=c_{r}\left(\mathbf{x}+\mathbf{1}_{\{r, s\}}\right)+c_{s}\left(\mathbf{x}+\mathbf{1}_{s}\right)=\alpha .
\end{aligned}
$$




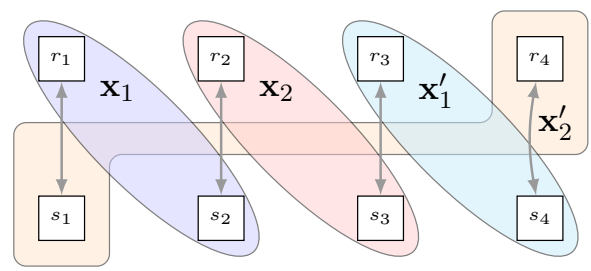

Figure 3: Game constructed for the proof of Lemma 3, respectively. Each clique represents a copy of the resource set (resources other than $r, s$ and dummy players are omitted). Player 1 chooses among strategies $\mathbf{x}_{1}$ and $\mathbf{x}_{1}^{\prime}$, player 2 chooses among strategies $\mathbf{x}_{2}$ and $\mathbf{x}_{2}^{\prime}$.

Similarly, one can verify that

$$
\begin{aligned}
& \pi_{2}\left(x^{(1,1)}\right)=\pi_{2}\left(x^{(2,2)}\right)=\pi_{1}\left(x^{(1,2)}\right)=\pi_{1}\left(x^{(2,1)}\right)=\alpha \text { and } \\
& \pi_{1}\left(x^{(1,1)}\right)=\pi_{1}\left(x^{(2,2)}\right)=\pi_{2}\left(x^{(1,2)}\right)=\pi_{2}\left(x^{(2,1)}\right)=\beta .
\end{aligned}
$$

Hence the above game is $(\alpha, \beta)$-symmetric for players 1 and 2 . Since $\mathcal{C}$ is consistent, the thus defined game has a pure Nash equilibrium and we conclude $\alpha=\beta$ by Lemma 2, which completes the proof of the lemma.

The following two lemmas establish that the discrete Hessian of each $c_{r}$ for $r \in[m]$ must be diagonal. For the proof of these two lemmas, we use the symmetry of the Jacobian shown in Lemma 3 together with suitably constructed $(\alpha, \beta)$-symmetric games. More specifically, the first lemma shows that for a consistent cost function $\mathbf{c}$ the discrete difference

$$
c_{r}\left(\mathbf{x}+\mathbf{1}_{s}\right)-c_{r}(\mathbf{x})
$$

is invariant under ( $i$ ) adding $\mathbf{1}_{r}$ to both arguments, or (ii) adding $\mathbf{1}_{s}$ to both arguments of the discrete difference.

Lemma 4. Let $\mathcal{C}$ be closed under composition and consistent for unweighted resource graph games. Then, for all $\mathbf{c} \in \mathcal{C}, \mathbf{c}: \mathbb{Z}_{\geq 0}^{m} \rightarrow \mathbb{R}^{m}$ with $m \in \mathbb{N}$, the following two functional equations are satisfied for all $r, s \in[m]$ with $r \neq s$ and all $\mathbf{x} \in \mathbb{Z}_{\geq 0}^{R}$ with $x_{r}>0$ :

(i) $c_{r}\left(\mathbf{x}+\mathbf{1}_{s}\right)-c_{r}(\mathbf{x})=c_{r}\left(\mathbf{x}+\mathbf{1}_{\{r, s\}}\right)-c_{r}\left(\mathbf{x}+\mathbf{1}_{r}\right)$ and

(ii) $c_{r}\left(\mathbf{x}+\mathbf{1}_{s}\right)-c_{r}(\mathbf{x})=c_{r}\left(\mathbf{x}+2 \cdot \mathbf{1}_{s}\right)-c_{r}\left(\mathbf{x}+\mathbf{1}_{s}\right)$.

Proof. Let $\mathbf{c} \in \mathcal{C}, \mathbf{c}: \mathbb{Z}_{\geq 0}^{m} \rightarrow \mathbb{R}^{m}, r, s \in[m]$ with $r \neq s$, and $\mathbf{y} \in \mathbb{Z}_{\geq 0}^{m}$ with $y_{r}>0$ be arbitrary. Since $\mathcal{C}$ is closed under composition, the function $\mathbf{c}^{4}: \mathbb{Z}_{\geq 0}^{4 m} \rightarrow \mathbb{R}^{4 m}$ is also contained in $\mathcal{C}$. Consider the following game with $4 m$ resources and cost function $\mathbf{c}^{4}$. For $k \in[4]$ and $t \in[m]$, we denote the $k$-th copy of resource $t$ by $t_{k}$. For each original resource $t \in[m] \backslash\{r\}$, there are $y_{t}$ dummy players whose only strategy is $\sum_{k \in[4]} \mathbf{1}_{t_{k}}$. There also are $y_{r}-1$ dummy players for resource $r$ whose only strategy is $\sum_{k \in[4]} \mathbf{1}_{r_{k}}$. In addition there are two players 1 and 2 with strategy sets

$$
X_{1}=\left\{\mathbf{1}_{\left\{r_{1}, s_{1}, r_{2}\right\}}, \mathbf{1}_{\left\{r_{3}, r_{4}, s_{4}\right\}}\right\} \quad \text { and } \quad X_{2}=\left\{\mathbf{1}_{\left\{r_{1}, r_{3}, s_{3}\right\}}, \mathbf{1}_{\left\{r_{2}, s_{2}, r_{4}\right\}}\right\} .
$$




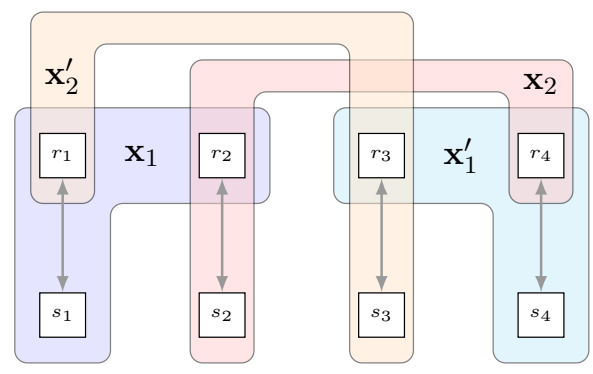

Figure 4: Game constructed for the proof of Lemma 4. Each clique represents a copy of the resource set (resources other than $r, s$ and dummy players are omitted). Player 1 chooses among strategies $\mathbf{x}_{1}$ and $\mathbf{x}_{1}^{\prime}$, player 2 chooses among strategies $\mathbf{x}_{2}$ and $\mathbf{x}_{2}^{\prime}$.

See Figure 4 for a depiction of the strategy space. Again, this game has only four strategy profiles

$$
\begin{array}{ll}
x^{(1,1)}=\left(\mathbf{1}_{\left\{r_{1}, s_{1}, r_{2}\right\}}, \mathbf{1}_{\left\{r_{1}, r_{3}, s_{3}\right\}}, x_{-\{1,2\}}\right), & x^{(1,2)}=\left(\mathbf{1}_{\left\{r_{1}, s_{1}, r_{2}\right\}}, \mathbf{1}_{\left\{r_{2}, s_{2}, r_{4}\right\}}, x_{-\{1,2\}}\right), \\
x^{(2,1)}=\left(\mathbf{1}_{\left\{r_{3}, r_{4}, s_{4}\right\}}, \mathbf{1}_{\left\{r_{1}, r_{3}, s_{3}\right\}}, x_{-\{1,2\}}\right), & x^{(2,2)}=\left(\mathbf{1}_{\left\{r_{3}, r_{4}, s_{4}\right\}}, \mathbf{1}_{\left\{r_{2}, s_{2}, r_{4}\right\}}, x_{-\{1,2\}}\right),
\end{array}
$$

where we again denote by $x_{-\{1,2\}}$ the unique strategy profile of all dummy players. We define

$$
\begin{aligned}
& \alpha=c_{r}\left(\mathbf{y}+\mathbf{1}_{\{r, s\}}\right)+c_{s}\left(\mathbf{y}+\mathbf{1}_{\{r, s\}}\right)+c_{r}(\mathbf{y}) \text { and } \\
& \beta=c_{r}\left(\mathbf{y}+\mathbf{1}_{s}\right)+c_{s}\left(\mathbf{y}+\mathbf{1}_{s}\right)+c_{r}\left(\mathbf{y}+\mathbf{1}_{\{r, s\}}\right) .
\end{aligned}
$$

Recalling the that there are $y_{r}-1$ dummy players on $r_{k}$ and $y_{s}$ dummy players on $s_{k}$ for $k \in[4]$ by construction, we observe that $x_{r_{1}}^{(1,1)}=y_{r}+1, x_{s_{1}}^{(1,1)}=y_{s}+1, x_{r_{2}}^{(1,1)}=y_{r}$, $x_{s 2}^{(1,1)}=y_{s}, x_{r_{3}}^{(1,1)}=y_{r}$, and $x_{s 3}^{(1,1)}=y_{s}+1$. Hence, we obtain

$$
\begin{aligned}
\pi_{1}\left(x^{(1,1)}\right) & =c_{r_{1}}^{4}\left(\mathbf{x}^{(1,1)}\right)+c_{s_{1}}^{4}\left(\mathbf{x}^{(1,1)}\right)+c_{r_{2}}^{4}\left(\mathbf{x}^{(1,1)}\right) \\
& =c_{r}\left(\mathbf{y}+\mathbf{1}_{\{r, s\}}\right)+c_{s}\left(\mathbf{y}+\mathbf{1}_{\{r, s\}}\right)+c_{r}(\mathbf{y})=\alpha, \\
\pi_{2}\left(x^{(1,1)}\right) & =c_{r_{1}}^{4}\left(\mathbf{x}^{(1,1)}\right)+c_{r_{3}}^{4}\left(\mathbf{x}^{(1,1)}\right)+c_{s_{3}}^{4}\left(\mathbf{x}^{(1,1)}\right) \\
& =c_{r}\left(\mathbf{y}+\mathbf{1}_{\{r, s\}}\right)+c_{r}\left(\mathbf{y}+\mathbf{1}_{s}\right)+c_{s}\left(\mathbf{y}+\mathbf{1}_{s}\right)=\beta .
\end{aligned}
$$

Similarly, we verify that

$$
\begin{aligned}
& \pi_{1}\left(x^{(1,1)}\right)=\pi_{1}\left(x^{(2,2)}\right)=\pi_{2}\left(x^{(1,2)}\right)=\pi_{2}\left(x^{(2,1)}\right)=\alpha \text { and } \\
& \pi_{2}\left(x^{(1,1)}\right)=\pi_{2}\left(x^{(2,2)}\right)=\pi_{1}\left(x^{(1,2)}\right)=\pi_{1}\left(x^{(2,1)}\right)=\beta .
\end{aligned}
$$

Hence the above game is $(\alpha, \beta)$-symmetric for players 1 and 2 .

By consistency of $\mathcal{C}$, the game must have a pure Nash equilibrium and thus $\alpha=\beta$ by Lemma 2. Subtracting the first and third term of $\alpha$ and the second term of $\beta$ on both sides yields

$$
c_{s}\left(\mathbf{y}+\mathbf{1}_{\{r, s\}}\right)-c_{s}\left(\mathbf{y}+\mathbf{1}_{s}\right)=c_{r}\left(\mathbf{y}+\mathbf{1}_{s}\right)-c_{r}(\mathbf{y}) .
$$


Applying Lemma 3 to the left-hand side of (7) yields

$$
c_{r}\left(\mathbf{y}+\mathbf{1}_{\{r, s\}}\right)-c_{r}\left(\mathbf{y}+\mathbf{1}_{r}\right)=c_{r}\left(\mathbf{y}+\mathbf{1}_{s}\right)-c_{r}(\mathbf{y}),
$$

which proves (i) when choosing $\mathbf{y}=\mathbf{x}$.

Applying Lemma 3 with $\mathbf{x}=\mathbf{y}-\mathbf{1}_{r}$ to the right-hand side of (7) instead, yields

$$
c_{s}\left(\mathbf{y}+\mathbf{1}_{\{r, s\}}\right)-c_{s}\left(\mathbf{y}+\mathbf{1}_{s}\right)=c_{s}\left(\mathbf{y}+\mathbf{1}_{s}\right)-c_{s}\left(\mathbf{y}-\mathbf{1}_{r}+\mathbf{1}_{s}\right) .
$$

This is equivalent to (ii) with the roles of $r$ and $s$ swapped, when choosing $\mathbf{y}=\mathbf{x}+\mathbf{1}_{r}-\mathbf{1}_{s}$.

The next lemma shows that for a consistent cost function $\mathbf{c}$ the discrete difference

$$
c_{r}\left(\mathbf{x}+\mathbf{1}_{s}\right)-c_{r}(\mathbf{x})
$$

is also invariant under adding $\mathbf{1}_{t}$ with $t \notin\{r, s\}$ to both arguments of the discrete difference.

Lemma 5. Let $\mathcal{C}$ be closed under composition and consistent for unweighted resource graph games. Then, for all $\mathbf{c} \in \mathcal{C}, \mathbf{c}: \mathbb{Z}_{\geq 0}^{m} \rightarrow \mathbb{R}^{m}$ with $m \in \mathbb{N}$ we have

$$
c_{r}\left(\mathbf{x}+\mathbf{1}_{s}\right)-c_{r}(\mathbf{x})=c_{r}\left(\mathbf{x}+\mathbf{1}_{\{s, t\}}\right)-c_{r}\left(\mathbf{x}+\mathbf{1}_{t}\right)
$$

for all $r, s, t \in[m]$ with $r, s, t$ pairwise distinct and all $\mathbf{x} \in \mathbb{Z}_{\geq 0}^{m}$ with $x_{r}>0$.

Proof. Let $\mathbf{c} \in \mathcal{C}, \mathbf{c}: \mathbb{Z}_{\geq 0}^{m} \rightarrow \mathbb{R}^{m}, m \in \mathbb{N}$ be arbitrary, let $r, s, t \in[m]$ be pairwise distinct, and let $\mathbf{x} \in \mathbb{Z}_{\geq 0}^{m}$ with $x_{r}>0$. Let $\mathbf{x}^{\prime}=\mathbf{x}-\mathbf{1}_{r}$. Consider the following game with $4 m$ resources and cost function $\mathbf{c}^{4}$. For $k \in[4]$ and $u \in[m]$, we denote the $k$-th copy of resource $u$ by $u_{k}$. For each resource $u \in[m]$, there are $x_{u}^{\prime}$ dummy players whose only strategy is $\sum_{k \in[4]} \mathbf{1}_{u_{k}}$. In addition there are two players 1 and 2 with strategy sets

$$
X_{1}=\left\{\mathbf{1}_{\left\{r_{1}, s_{2}, t_{2}\right\}}, \mathbf{1}_{\left\{s_{3}, t_{3}, r_{4}\right\}}\right\} \quad \text { and } \quad X_{2}=\left\{\mathbf{1}_{\left\{s_{1}, t_{1}, r_{3}\right\}}, \mathbf{1}_{\left\{r_{2}, s_{4}, t_{4}\right\}}\right\} .
$$

See Figure 5 for a depiction of the strategy space. As in the proof of the two previous lemmas, the game has only four strategy profiles:

$$
\begin{array}{ll}
\mathbf{x}^{(1,1)}=\left(\mathbf{1}_{\left\{r_{1}, s_{2}, t_{2}\right\}}, \mathbf{1}_{\left\{s_{1}, t_{1}, r_{3}\right\}}, x_{-\{1,2\}}\right), & \mathbf{x}^{(1,2)}=\left(\mathbf{1}_{\left\{r_{1}, s_{2}, t_{2}\right\}}, \mathbf{1}_{\left\{r_{2}, s_{4}, t_{4}\right\}}, x_{-\{1,2\}}\right), \\
\mathbf{x}^{(2,1)}=\left(\mathbf{1}_{\left\{s_{3}, t_{3}, r_{4}\right\}}, \mathbf{1}_{\left\{s_{1}, t_{1}, r_{3}\right\}}, x_{-\{1,2\}}\right), & \mathbf{x}^{(2,2)}=\left(\mathbf{1}_{\left\{s_{3}, t_{3}, r_{4}\right\}}, \mathbf{1}_{\left\{r_{2}, s_{4}, t_{4}\right\}}, x_{-\{1,2\}}\right),
\end{array}
$$

where $x_{-\{1,2\}}$ denotes the unique strategy profile of the dummy players. Along the same lines as before, one can check that

$$
\begin{aligned}
& \pi_{1}\left(\mathbf{x}^{(1,1)}\right)=\pi_{1}\left(\mathbf{x}^{(2,2)}\right)=\pi_{2}\left(\mathbf{x}^{(1,2)}\right)=\pi_{2}\left(\mathbf{x}^{(2,1)}\right)=\alpha \text { and } \\
& \pi_{2}\left(\mathbf{x}^{(1,1)}\right)=\pi_{2}\left(\mathbf{x}^{(2,2)}\right)=\pi_{1}\left(\mathbf{x}^{(1,2)}\right)=\pi_{1}\left(\mathbf{x}^{(2,1)}\right)=\beta,
\end{aligned}
$$

where

$$
\begin{aligned}
\alpha & =c_{r}\left(\mathbf{x}^{\prime}+\mathbf{1}_{\{r, s, t\}}\right)+c_{s}\left(\mathbf{x}^{\prime}+\mathbf{1}_{\{s, t\}}\right)+c_{t}\left(\mathbf{x}^{\prime}+\mathbf{1}_{\{s, t\}}\right) \text { and } \\
\beta & =c_{r}\left(\mathbf{x}^{\prime}+\mathbf{1}_{r}\right)+c_{s}\left(\mathbf{x}^{\prime}+\mathbf{1}_{\{r, s, t\}}\right)+c_{t}\left(\mathbf{x}^{\prime}+\mathbf{1}_{\{r, s, t\}}\right)
\end{aligned}
$$




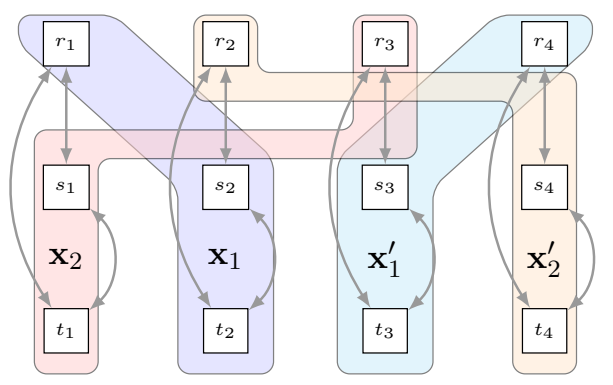

Figure 5: Game constructed for the proof of Lemma 5. Each clique represents a copy of the resource set (resources other than $r, s, t$ and dummy players are omitted). Player 1 chooses among strategies $\mathbf{x}_{1}$ and $\mathbf{x}_{1}^{\prime}$, player 2 chooses among strategies $\mathbf{x}_{2}$ and $\mathbf{x}_{2}^{\prime}$.

Hence, the game is $(\alpha, \beta)$-symmetric for players 1 and 2 .

By consistency of $\mathcal{C}$ the game must have a pure Nash equilibrium and thus $\alpha=\beta$ by Lemma 2. By subtracting the second term of $\alpha$ and the first and third term of $\beta$ from both sides we obtain

$$
\begin{aligned}
& c_{r}\left(\mathbf{x}^{\prime}+\mathbf{1}_{\{r, s, t\}}\right)-\underbrace{\left(c_{t}\left(\mathbf{x}^{\prime}+\mathbf{1}_{\{r, s, t\}}\right)-c_{t}\left(\mathbf{x}^{\prime}+\mathbf{1}_{\{s, t\}}\right)\right)}_{=c_{r}\left(\mathbf{x}^{\prime}+\mathbf{1}_{\{r, s, t\}}\right)-c_{r}\left(\mathbf{x}^{\prime}+\mathbf{1}_{\{r, s\}}\right)}-c_{r}\left(\mathbf{x}^{\prime}+\mathbf{1}_{r}\right) \\
= & \underbrace{c_{s}\left(\mathbf{x}^{\prime}+\mathbf{1}_{\{r, s, t\}}\right)-c_{s}\left(\mathbf{x}^{\prime}+\mathbf{1}_{\{s, t\}}\right)}_{=c_{r}\left(\mathbf{x}^{\prime}+\mathbf{1}_{\{r, s, t\}}\right)-c_{r}\left(\mathbf{x}^{\prime}+\mathbf{1}_{\{r, t\}}\right)} .
\end{aligned}
$$

Applying the identities indicated above, which follow from Lemma 3, and then using $\mathbf{x}^{\prime}=$ $\mathbf{x}-\mathbf{1}_{r}$ yields $c_{r}\left(\mathbf{x}+\mathbf{1}_{s}\right)-c_{r}(\mathbf{x})=c_{r}\left(\mathbf{x}+\mathbf{1}_{\{s, t\}}\right)-c_{r}\left(\mathbf{x}+\mathbf{1}_{t}\right)$.

Given the form of the discrete Hessian established in Lemmas 4 and 5, we conclude now that the influence of the load on a resource $s$ on the cost of some other resource $r$ must be linear. This is formalized in the following lemma, which follows by inductively applying our previous results.

Lemma 6. Let $\mathcal{C}$ be closed under composition and consistent for unweighted resource graph games. Then, for all $\mathbf{c} \in \mathcal{C}, \mathbf{c}: \mathbb{Z}_{\geq 0}^{m} \rightarrow \mathbb{R}^{m}$ with $m \in \mathbb{N}$ we have

$$
c_{r}\left(\mathbf{x}+\mathbf{1}_{s}\right)-c_{r}(\mathbf{x})=c_{r}\left(\mathbf{y}+\mathbf{1}_{s}\right)-c_{r}(\mathbf{y})
$$

for all $r, s \in[m]$ with $r \neq s$ and all $\mathbf{x}, \mathbf{y} \in \mathbb{Z}_{\geq 0}^{m}$ with $x_{r}, y_{r}>0$.

Proof. Let $\mathbf{c} \in \mathcal{C}, \mathbf{c}: \mathbb{Z}_{\geq 0}^{m} \rightarrow \mathbb{R}^{m}, r, s \in[m]$ with $r \neq s$, and $\mathbf{x}, \mathbf{y} \in \mathbb{Z}_{\geq 0}^{m}$ with $x_{r}, y_{r}>0$ be arbitrary. We show the lemma by induction on $k=\sum_{r^{\prime} \in R}\left|x_{r^{\prime}}-y_{r^{\prime}}\right|$. If $k=0$, then $x=y$ and the claim is trivially fulfilled. Thus assume $k>0$. Without loss of generality, there is $t \in R$ with $x_{t}>y_{t}$. Let $\mathbf{x}^{\prime}:=\mathbf{x}-\mathbf{1}_{t}$. Note that $x_{r}^{\prime}>0$. Indeed, if $r=t$ then $x_{r}^{\prime} \geq y_{r}>0$. If, on the other hand, $r \neq t$, then $x_{r}^{\prime}=x_{r}>0$. Thus, Lemma 4 and Lemma 5 can be applied to $\mathbf{x}^{\prime}$. We distinguish three cases for $t$.

Case $t=r$ : In this case, $\mathbf{x}=\mathbf{x}^{\prime}+\mathbf{1}_{r}$. We obtain

$$
c_{r}\left(\mathbf{x}+\mathbf{1}_{s}\right)-c_{r}(\mathbf{x})=c_{r}\left(\mathbf{x}^{\prime}+\mathbf{1}_{\{r, s\}}\right)-c_{r}\left(\mathbf{x}^{\prime}+\mathbf{1}_{r}\right)=c_{r}\left(\mathbf{x}^{\prime}+\mathbf{1}_{s}\right)-c_{r}\left(\mathbf{x}^{\prime}\right) .
$$

where the second equality follows from Lemma 4. 
Case $t=s:$ In this case, $\mathbf{x}=\mathbf{x}^{\prime}+\mathbf{1}_{s}$. We obtain

$$
c_{r}\left(\mathbf{x}+\mathbf{1}_{s}\right)-c_{r}(\mathbf{x})=c_{r}\left(\mathbf{x}^{\prime}+2 \cdot \mathbf{1}_{s}\right)-c_{r}\left(\mathbf{x}^{\prime}+\mathbf{1}_{s}\right)=c_{r}\left(\mathbf{x}^{\prime}+\mathbf{1}_{s}\right)-c_{r}\left(\mathbf{x}^{\prime}\right) .
$$

where the second equality follows from Lemma 4.

Case $t \in R \backslash\{r, s\}:$ In this case, $\mathbf{x}=\mathbf{x}^{\prime}+\mathbf{1}_{t}$. We obtain

$$
c_{r}\left(\mathbf{x}+\mathbf{1}_{s}\right)-c_{r}(\mathbf{x})=c_{r}\left(\mathbf{x}^{\prime}+\mathbf{1}_{\{s, t\}}\right)-c_{r}\left(\mathbf{x}^{\prime}+\mathbf{1}_{t}\right)=c_{r}\left(\mathbf{x}^{\prime}+\mathbf{1}_{s}\right)-c_{r}\left(\mathbf{x}^{\prime}\right) .
$$

where the second equality follows from Lemma 5 .

In either case, $c_{r}\left(\mathbf{x}+\mathbf{1}_{s}\right)-c_{r}(\mathbf{x})=c_{r}\left(\mathbf{x}^{\prime}+\mathbf{1}_{s}\right)-c_{r}\left(\mathbf{x}^{\prime}\right)$, which is equal to $c_{r}\left(\mathbf{y}+\mathbf{1}_{s}\right)-c_{r}(\mathbf{y})$ by the induction hypothesis because $\sum_{r^{\prime} \in R}\left|x_{r^{\prime}}^{\prime}-y_{r^{\prime}}\right|<k$.

Combining Lemmas 3 and 6, we observe that the interaction effects of distinct resources in the cost function c must be linear and symmetric and thus condition (6) is indeed necessary for consistency. This is formalized in the following lemma, which completes the proof of Theorem 1.

Lemma 7. Let $\mathcal{C}$ be closed under composition and consistent for unweighted resource graph games. Then, for all $\mathbf{c} \in \mathcal{C}, \mathbf{c}: \mathbb{Z}_{\geq 0}^{m} \rightarrow \mathbb{R}^{m}$ with $m \in \mathbb{N}$ there are $m$ functions $f_{1}, \ldots, f_{m}$ : $\mathbb{Z}_{\geq 0} \rightarrow \mathbb{R}$ and a symmetric matrix $\mathbf{A} \in \mathbb{R}^{m \times m}$ such that $\mathbf{c}$ is equivalent to the function $\mathbf{c}^{\prime}$ defined by $\mathbf{c}^{\prime}(\mathbf{x})=\left(f_{1}\left(x_{1}\right), \ldots, f_{m}\left(x_{m}\right)\right)^{\top}+\mathbf{A} \mathbf{x}$ for all $\mathbf{x} \in \mathbb{Z}_{\geq 0}^{R}$.

Proof. For $r \in R$ define $f_{r}: \mathbb{Z}_{\geq 0} \rightarrow \mathbb{R}$ by $f_{r}(x)=c_{r}\left(x \cdot \mathbf{1}_{r}\right)$ for all $x \in \mathbb{Z}_{\geq 0}$. The matrix $\mathbf{A} \in \mathbb{R}^{m \times m}=\left(a_{r, s}\right)_{r, s \in[m]}$ is defined as

$$
a_{r, s}= \begin{cases}c_{r}\left(\mathbf{1}_{\{r, s\}}\right)-c_{r}\left(\mathbf{1}_{r}\right) & \text { if } r \neq s \\ 0 & \text { otherwise. }\end{cases}
$$

Note that $a_{r, s}=a_{s, r}$ and, hence $\mathbf{A}$ is symmetric by Lemma 3 .

Let $\mathbf{x} \in \mathbb{Z}_{\geq 0}^{m}$ and $r \in[m]$ with $x_{r}>0$. We denote by $\mathbf{A}_{r, \text {. }}$ the $r$-th row of the matrix $\mathbf{A}$. We show that $c_{r}(\mathbf{x})=f_{r}\left(x_{r}\right)+A_{r,} \mathbf{x}$ by induction on $k=\sum_{s \in R \backslash\{r\}} x_{s}$. For $k=0$ the claim is true by definition of $f_{r}$. Thus assume $k>0$ and let $s \in R \backslash\{r\}$ with $x_{s}>0$. We obtain

$$
\begin{aligned}
c_{r}(\mathbf{x}) & =c_{r}\left(\mathbf{x}-\mathbf{1}_{s}\right)+c_{r}\left(\mathbf{1}_{\{r, s\}}\right)-c_{r}\left(\mathbf{1}_{r}\right) \\
& =f_{r}\left(x_{r}\right)+\mathbf{A}_{r, \cdot}\left(\mathbf{x}-\mathbf{1}_{s}\right)+a_{r, s} \\
& =f_{r}\left(x_{r}\right)+\mathbf{A}_{r, \cdot} \mathbf{x}
\end{aligned}
$$

where the first identity follows from Lemma 6 and the second identity follows from the induction hypothesis and the definition of $a_{r, s}$.

\section{Resource Graph Games with Weighted Players}

In this section, we establish necessary and sufficient conditions for consistency when each player $i \in N$ imposes a weight $w_{i} \in \mathbb{R}_{\geq 0}$ on the resources in their strategy. The characterization reveals two possible cases: A consistent set of cost functions either contains only affine functions with a symmetric Jacobian, or the cost functions of individual resources are exponential and separable (i.e., there is no interaction among distinct resources). 
Theorem 2. Let $\mathcal{C}$ be a set of continuous cost functions that is closed under composition. Then $\mathcal{C}$ is consistent for weighted resource graph games if and only if one of the following two statements is fulfilled:

(i) For each $\mathbf{c} \in \mathcal{C}$ with $\mathbf{c}: \mathbb{R}_{>0}^{m} \rightarrow \mathbb{R}^{m}$ for some $m \in \mathbb{N}$ there is a symmetric matrix $\mathbf{A} \in \mathbb{R}^{m \times m}$ and a vector $\mathbf{b} \in \mathbb{R}^{m}$ such that $\mathbf{c}(\mathbf{x})=\mathbf{A} \mathbf{x}+\mathbf{b}$.

(ii) There is $\phi \in \mathbb{R}$ such that for all $\mathbf{c} \in \mathcal{C}$ with $\mathbf{c}: \mathbb{R}_{\geq 0}^{m} \rightarrow \mathbb{R}^{m}$ there are $\mathbf{a}, \mathbf{b} \in \mathbb{R}^{m}$ such that $c_{r}(\mathbf{x})=a_{r} \exp \left(\phi x_{r}\right)+b_{r}$ for all $r \in[m]$ and all $\mathbf{x} \in \mathbb{R}_{\geq 0}^{m}$.

The two distinct cases arise due to the fact that weighted congestion games are a special case of weighted resource graph games, namely where the cost of each resource $r$ depends on the load of $r$ only, i.e., $B_{r}=\{r\}$ for all $r \in R$. For these games, Harks and Klimm (2012) provided a characterization that shows that consistent sets contain only affine or only exponential cost functions. In the following, we prove the sufficiency and necessity of either of the two conditions.

\subsection{Proof of Theorem 2: (i) or (ii) $\Rightarrow$ Consistency of $\mathcal{C}$}

We show sufficiency of conditions (i) or (i) in Theorem 2, respectively, for the consistency of $\mathcal{C}$. If condition 2 is fulfilled, then any weighted resource graph game with cost function $\mathbf{c} \in \mathcal{C}$ is a weighted congestion game with exponential costs. For these games, the existence of pure Nash equilibria has been established by Theorem 5.1 of Harks and Klimm (2012). It is therefore sufficient to show that condition 1 of Theorem 2 is also sufficient for consistency. The following lemma establishes the sufficiency of condition 1, following the same lines as the proof of Lemma 1.

Lemma 8. Let $G$ be a weighted resource graph game on $m$ resources with cost function $\mathbf{c}: \mathbb{R}_{\geq 0}^{m} \rightarrow \mathbb{R}^{m}$ given by $\mathbf{c}(\mathbf{x})=\mathbf{A x}+\mathbf{b}$, where $\mathbf{A} \in \mathbb{R}^{m \times m}$ is a symmetric matrix and $\mathbf{b} \in \mathbb{R}^{m}$ is a vector. Then $G$ has a pure Nash equilibrium.

Proof. Fix an arbitrary weighted resource graph game $G$ whose cost is determined by c $\in \mathcal{C}$ and an arbitrary strategy profile $x \in X$. As in the proof of Lemma 1 , let $P(x)$ be the sum of the private costs of the players when adding them to the game in order $1, \ldots, n$. We again write $\mathbf{x}_{\leq i}=\sum_{j \in N: j \leq i} \mathbf{x}_{j}$ for the load vector of the players up to $i$. We then obtain

$$
P(x)=\sum_{i \in N} \mathbf{x}_{i}^{\top}\left[\mathbf{A} \mathbf{x}_{\leq i}+\mathbf{b}\right] .
$$

Similarly to the proof of Theorem 1, we calculate

$$
\begin{aligned}
\sum_{i \in N} \mathbf{x}_{i}^{\top} \mathbf{A} \mathbf{x}_{\leq i} & =\sum_{i \in N} \mathbf{x}_{i}^{\top} \mathbf{A}\left(\sum_{j \in N: j \leq i} \mathbf{x}_{j}\right) \\
& =\frac{1}{2} \sum_{i \in N} \sum_{j \in N} \mathbf{x}_{i}^{\top} \mathbf{A} \mathbf{x}_{j}+\frac{1}{2} \sum_{i \in N} \mathbf{x}_{i}^{\top} \mathbf{A} \mathbf{x}_{i} \\
& =\frac{1}{2} \mathbf{x}^{\top} \mathbf{A} \mathbf{x}+\frac{1}{2} \sum_{i \in N} \mathbf{x}_{i}^{\top} \mathbf{A} \mathbf{x}_{i},
\end{aligned}
$$


as in the unweighted case where we again used the symmetry of $\mathbf{A}$. We obtain

$$
P(x)=\frac{1}{2} \mathbf{x}^{\top} \mathbf{A} \mathbf{x}+\frac{1}{2} \sum_{i \in N} \mathbf{x}_{i}^{\top}\left[\mathbf{A} \mathbf{x}_{i}+2 \mathbf{b}\right] .
$$

This shows that $P$ is independent of the ordering of the players and the remainder of the proof is similar to Lemma 1.

\subsection{Proof of Theorem 2: Consistency of $\mathcal{C} \Rightarrow$ (i) or (ii)}

We now prove the necessity of the conditions given in Theorem 2. By a slight adaptation of the constructions in Section 3, we obtain the following stronger version of Lemmas 3 and 6 for cost functions that are consistent for weighted players.

Lemma 9. Let $\mathcal{C}$ be closed under composition and consistent for weighted resource graph games. Then, for all $\mathbf{c} \in \mathcal{C}, \mathbf{c}: \mathbb{R}_{\geq 0}^{m} \rightarrow \mathbb{R}^{m}$ with $m \in \mathbb{N}$ the following functional equations are satisfied:

(i) $c_{r}\left(\mathbf{x}+\varepsilon \cdot \mathbf{1}_{\{r, s\}}\right)-c_{r}\left(\mathbf{x}+\varepsilon \cdot \mathbf{1}_{r}\right)=c_{s}\left(\mathbf{x}+\varepsilon \cdot \mathbf{1}_{\{r, s\}}\right)-c_{s}\left(\mathbf{x}+\varepsilon \cdot \mathbf{1}_{s}\right)$ for all $r, s \in R$ and all $\mathbf{x} \in \mathbb{R}_{\geq 0}^{m}$ and all $\varepsilon>0$ and

(ii) $c_{r}\left(\mathbf{x}+\varepsilon \cdot \mathbf{1}_{s}\right)-c_{r}(\mathbf{x})=c_{r}\left(\mathbf{y}+\varepsilon \cdot \mathbf{1}_{s}\right)-c_{r}(\mathbf{y})$ for all $r, s \in R$ with $r \neq s$ and all $\mathbf{x}, \mathbf{y} \in \mathbb{R}_{\geq 0}^{m}$ with $x_{r}, y_{r}>0$ and all $\varepsilon>0$.

Proof (Sketch). We follow the same constructions used to establish Lemmas 3, 4, 5, and 6. However, we set $w_{1}=w_{2}=\varepsilon$ and adjust the weights of the dummy players for each resource such that the load on the resource equals the corresponding coordinate of $\mathbf{x}$.

The following lemma follows from the characterization of consistent functions for weighted congestion games with separable cost functions due to Harks and Klimm (2012).

Lemma 10. Let $\mathcal{C}$ be a set of continuous functions that is closed under composition and consistent for weighted resource graph games. Then, for all $\mathbf{c} \in \mathcal{C}, \mathbf{c}: \mathbb{R}_{\geq 0}^{m} \rightarrow \mathbb{R}^{m}$ with $m \in \mathbb{N}$ one of the following statements is true:

(i) For all $S \subseteq R$ and all $\mathbf{z} \in \mathbb{R}_{\geq 0}^{m}$ there are $a_{S, \mathbf{z}}, b_{S, \mathbf{z}} \in \mathbb{R}$ such that $\sum_{r \in S} c_{r}\left(\mathbf{z}+\lambda \mathbf{1}_{S}\right)=$ $a_{S, \mathbf{z}} \lambda+b_{S, \mathbf{z}}$ for all $\lambda \geq 0$.

(ii) There is $\phi \in \mathbb{R}$ such that for all $S \subseteq R$ and all $\mathbf{z} \in \mathbb{R}_{\geq 0}^{m}$ there are $a_{S, \mathbf{z}}, b_{S, \mathbf{z}} \in \mathbb{R}$ such that $\sum_{r \in S} c_{r}\left(\mathbf{z}+\lambda \mathbf{1}_{S}\right)=a_{S, \mathbf{z}} \exp (\phi \lambda)+b_{S, \mathbf{z}}$ for all $\lambda \geq 0$.

Proof. Let $\mathbf{c} \in \mathcal{C}, \mathbf{c}: \mathbb{R}_{>0}^{m} \rightarrow \mathbb{R}^{m}$ with $m \in \mathbb{N}$ be arbitrary. For $S \subseteq R$ and $\mathbf{z} \in \mathbb{R}_{>0}^{m}$ define $c_{S, \mathbf{z}}: \mathbb{R}_{\geq 0} \rightarrow \mathbb{R}$ by $c_{S, \mathbf{z}}(\lambda)=\sum_{r \in S} c_{r}\left(\mathbf{z}+\lambda \mathbf{1}_{S}\right)$. Let $\mathcal{C}^{\prime}=\left\{c_{S, \mathbf{z}}: S \subseteq R, \mathbf{z} \in \mathbb{R}^{m}\right\}$ be the set of all functions arising in this way. We show that any weighted congestion game with separable cost functions on $k$ resources where each resource has a cost function $c^{\prime} \in \mathcal{C}^{\prime}$ is isomorphic to a weighted resource graph game with cost function $\mathbf{c}^{k}$ on $\mathrm{km}$ resources. Since $\mathcal{C}$ is closed under composition, the function $\mathbf{c}^{k}$ is contained in $\mathcal{C}$, and, hence, consistency of $\mathcal{C}$ for weighted resource graph games implies the consistency of $\mathcal{C}^{\prime}$ for weighted congestion games. A set of continuous functions is consistent for weighted congestion games if and 
only if it contains only affine functions (as described in case (i) of the lemma) or it contains only exponential functions (as described in case (ii) of the lemma) (Harks \& Klimm, 2012, Theorem 5.1). Hence the lemma follows from the following construction.

Consider any weighted congestion game $G^{\prime}$ with arbitrary player set $N^{\prime}$, weights $w_{i}^{\prime}$ for each $i \in N^{\prime}$, strategies $X_{i}^{\prime}=\left\{w_{i} \cdot \mathbf{x}_{i}: \mathbf{x}_{i} \in Y_{i}\right\}$ with $Y_{i} \subseteq\{0,1\}^{k}$, and resource set $R^{\prime}=\left\{r_{1}^{\prime}, \ldots, r_{k}^{\prime}\right\}$ such that for all $r \in R^{\prime}$ the cost function $c_{r}^{\prime}: \mathbb{R}_{\geq 0} \rightarrow \mathbb{R}$ of resource $r$ is of the form $c_{r}^{\prime}=c_{S, \mathbf{z}}$ for some $S \subseteq R$ and $\mathbf{z} \in \mathbb{R}_{\geq 0}^{R}$. In what follows, we construct an isomorphic weighted resource graph game $G$ with player set $N, m k$ resources, and cost function $\mathbf{c}^{k}$. For $j \in[k]$ let $S_{j} \subseteq R$ and $\mathbf{z}_{j}=\left(z_{j, 1}, \ldots, z_{j, m}\right) \in \mathbb{R}_{\geq 0}^{m}$ be such that $c_{r_{j}}^{\prime}=c_{S_{j}, \mathbf{z}_{j}}$. We define $N=N^{\prime} \cup\{(r, j): r \in R, j \in[k]\}$, i.e., the set of players $N$ of $G$ contains the player set $N^{\prime}$ of the original congestion game plus $m k$ additional dummy players. Each dummy player $(r, j)$ can only play strategy $\mathbf{1}_{r_{j}}$ where $r_{j}$ is the $j$-th copy of resource $r \in[m]$. That dummy player has a weight $w_{(r, j)}=z_{j, r}$. Each normal player $i \in N^{\prime}$ has the same weight $w_{i}=w_{i}^{\prime}$ as in the original congestion game. For each strategy $\mathbf{x}_{i}^{\prime} \in X_{i}^{\prime}$ of player $i$ in the original congestion game, there is a strategy $\mathbf{x}_{i} \in X_{i}$ that arises from $\mathbf{x}_{i}^{\prime}$ by replacing each resource $r_{j} \in R^{\prime}$ by the set of resources $S_{j} \subseteq R$, i.e., $\mathbf{X}_{i}=\left\{\sum_{j \in[k]} x_{i, j}^{\prime} \mathbf{1}_{S_{j}}: \mathbf{x}_{i}^{\prime}=\left(x_{i, 1}^{\prime}, \ldots, x_{i, k}^{\prime}\right) \in X_{i}^{\prime}\right\}$. Thus, there is a one-to-one correspondence between strategy profiles $\mathbf{x}^{\prime}$ for $G^{\prime}$ and strategy profiles x of $G$ and it is easy to see that by construction, the private cost of player $i \in N^{\prime}$ is the same for $\mathbf{x}^{\prime}$ in $G^{\prime}$ and the corresponding profile $\mathbf{x}$ in $G$.

Equipped with Lemmas 9 and 10, we can show that the impact of the load of resource $s$ on the cost of resource $r$ needs to be linear and symmetric. In addition, the impact is non-existent if case (i) of Lemma 10 does not hold. This is formalized in the following lemma.

Lemma 11. Let $\mathcal{C}$ be a set of continuous functions that is closed under composition and consistent for weighted resource graph games. Then, for all $\mathbf{c} \in \mathcal{C}, \mathbf{c}: \mathbb{R}_{\geq 0}^{m} \rightarrow \mathbb{R}^{m}$ with $m \in \mathbb{N}$ and all $r, s \in[m]$ with $r \neq s$ there is $a_{r, s}=a_{s, r}$ such that

$$
c_{r}\left(\mathbf{z}+\lambda \mathbf{1}_{s}\right)-c_{r}(\mathbf{z})=a_{r, s} \lambda
$$

for all $\mathbf{z} \in \mathbb{R}_{\geq 0}^{m}$ with $z_{r}>0$ and all $\lambda \geq 0$. Moreover, if case (i) of Lemma 10 does not hold, then $a_{r, s}=0$ for all $r, s \in R$.

Proof. Let $\mathbf{c} \in \mathcal{C}, \mathbf{c}: \mathbb{R}_{\geq 0}^{m} \rightarrow \mathbb{R}^{m}$ with $m \in \mathbb{N}$ be arbitrary. Let $r, s \in[m]$ with $r \neq s$ and let $\mathbf{z} \in \mathbb{R}_{\geq 0}^{m}$ with $z_{r}>0$. Applying Lemma 9 (i) to rewrite $c_{s}\left(\mathbf{z}+\lambda \mathbf{1}_{\{r, s\}}\right)$ we obtain

$$
\begin{aligned}
& \underbrace{c_{r}\left(\mathbf{z}+\lambda \mathbf{1}_{\{r, s\}}\right)+c_{s}\left(\mathbf{z}+\lambda \mathbf{1}_{\{r, s\}}\right)}_{h_{0}(\lambda)} \\
& =c_{r}\left(\mathbf{z}+\lambda \mathbf{1}_{\{r, s\}}\right)+c_{r}\left(\mathbf{z}+\lambda \mathbf{1}_{\{r, s\}}\right)-c_{r}\left(\mathbf{z}+\lambda \mathbf{1}_{r}\right)+c_{s}\left(\mathbf{z}+\lambda \mathbf{1}_{s}\right) \\
& =\underbrace{c_{r}\left(\mathbf{z}+\lambda \mathbf{1}_{r}\right)}_{h_{1}(\lambda)}+\underbrace{c_{s}\left(\mathbf{z}+\lambda \mathbf{1}_{s}\right)}_{h_{2}(\lambda)}+2(\underbrace{c_{r}\left(\mathbf{z}+\lambda \mathbf{1}_{\{r, s\}}\right)-c_{r}\left(\mathbf{z}+\lambda \mathbf{1}_{r}\right)}_{h_{4}(\lambda)})
\end{aligned}
$$

for all $\lambda \geq 0$. We apply Lemma 10 to the expressions $h_{0}(\lambda), h_{1}(\lambda), h_{2}(\lambda)$ and distinguish two cases. 
If we are in case $(i)$ of Lemma 10, then all three expressions are affine functions of $\lambda$ and we conclude that also $h_{4}$ must be affine in $\lambda$, i.e., there are $a_{\mathbf{z}}, b_{\mathbf{z}} \in \mathbb{R}$ such that

$$
c_{r}\left(\mathbf{z}+\lambda \mathbf{1}_{\{r, s\}}\right)-c_{r}\left(\mathbf{z}+\lambda \mathbf{1}_{r}\right)=a_{\mathbf{z}} \lambda+b_{\mathbf{z}}
$$

By part (i) of Lemma 9, we observe that this equality also holds (for the same values of $a_{\mathbf{z}}$ and $b_{\mathbf{z}}$ ) when swapping the roles of $s$ and $r$. Applying part (ii) of Lemma 9, we observe that $a_{\mathbf{z}}$ and $b_{\mathbf{z}}$ are independent of $\mathbf{z}$. Letting $\lambda$ approach 0 , continuity of $c_{r}$ implies that $b_{\mathbf{z}}=0$.

If we are in case (ii) of Lemma 10, then all three expressions are exponential functions of the form $a \exp (\phi \lambda)+b$ for some $\phi \in \mathbb{R}$ and we conclude that also $h_{4}$ must be of this form, i.e., there is $a^{\prime}, b^{\prime} \in R$ such that $c_{r}\left(\mathbf{z}+\lambda \mathbf{1}_{\{r, s\}}\right)-c_{r}\left(\mathbf{z}+\lambda \mathbf{1}_{r}\right)=a^{\prime} \exp (\phi \lambda)+b^{\prime}$. Applying part (ii) of Lemma 9 , we conclude that $a^{\prime}=b^{\prime}=0$, thus proving the statement of the lemma for this case.

We are now ready to establish the necessity of condition 1 or 2 of Theorem 2, concluding the proof of the theorem.

Lemma 12. Let $\mathcal{C}$ be a set of continuous functions that is closed under composition and consistent for weighted resource graph games. Then, one of the following statements is true:

1. For each $\mathbf{c} \in \mathcal{C}$ with $\mathbf{c}: \mathbb{R}_{\geq 0}^{m} \rightarrow \mathbb{R}^{m}$ for some $m \in \mathbb{N}$ there is a symmetric matrix $\mathbf{A} \in \mathbb{R}^{m \times m}$ and a vector $\mathbf{b} \in \mathbb{R}^{m}$ such that $\mathbf{c}(\mathbf{x})=\mathbf{A} \mathbf{x}+\mathbf{b}$.

2. There is $\phi \in \mathbb{R}$ such that for all $\mathbf{c} \in \mathcal{C}$ with $\mathbf{c}: \mathbb{R}_{>0}^{m} \rightarrow \mathbb{R}^{m}$ there are $\mathbf{a}, \mathbf{b} \in \mathbb{R}^{m}$ such that $c_{r}(\mathbf{x})=a_{r} \exp \left(\phi x_{r}\right)+b_{r}$ for all $r \in[m]$ and all $\mathbf{x} \in \mathbb{R}_{\geq 0}^{m}$.

Proof. Let $\mathbf{c} \in \mathcal{C}, \mathbf{c}: \mathbb{R}_{\geq 0}^{m} \rightarrow \mathbb{R}^{m}$ with $m \in \mathbb{N}$ be arbitrary. By Lemma 11, for all $r, s \in[m]$, there is $a_{r, s}$ such that $c_{r}\left(\mathbf{z}+\lambda \mathbf{1}_{s}\right)-c_{r}(\mathbf{z})=a_{r, s} \lambda$ for all $\mathbf{z} \in \mathbb{R}_{\geq 0}^{m}$ with $z_{r}>0$ and all $\lambda \geq 0$. Let $r_{1}, \ldots, r_{m}$ be an arbitrary ordering of the resources in $R$ with $r_{m}=r$. Defining $\mathbf{x}^{(0)}=\mathbf{x}$ and $\mathbf{x}^{(i)}=\mathbf{x}^{(i-1)}-x_{r_{i}} \cdot \mathbf{1}_{r_{i}}$ for $i \in[m]$ we obtain

$$
\begin{aligned}
c_{r}(\mathbf{x}) & =c_{r}\left(x_{r} \cdot \mathbf{1}_{r}\right)+\sum_{i=1}^{m-1} c_{r}\left(\mathbf{x}^{(i-1)}\right)-c_{r}\left(\mathbf{x}^{(i)}\right) \\
& =c_{r}\left(x_{r} \cdot \mathbf{1}_{r}\right)+\sum_{s \in R \backslash\{r\}} a_{r, s}\left(x_{s}-1\right) .
\end{aligned}
$$

In case (i) of Lemma 10, we conclude that $c_{r}\left(x_{r} \cdot \mathbf{1}_{r}\right)$ is an affine function of $x_{r}$. This implies that there is a symmetric matrix $\mathbf{A} \in \mathbb{R}^{m \times m}$ and a vector $\mathbf{b} \in \mathbb{R}^{m}$ such that $c_{r}(\mathbf{x})=\mathbf{A}_{r,} \mathbf{x}+b_{r}$ for all $r \in R$ and all $\mathbf{x} \in \mathbb{R}_{\geq 0}^{m}$ with $x_{r}>0$. Since $\mathcal{C}$ is closed under composition, all functions $\mathbf{c} \in \mathcal{C}$ have this property thus we retrieve case 1 of Lemma 12 .

In case (ii) of Lemma 10, we conclude that $c_{r}\left(x_{r} \cdot \mathbf{1}_{r}\right)$ is an exponential function of $x_{r}$. By Lemma 11, we than have that $a_{r, s}=0$ and we thus obtain that $c_{r}(\mathbf{x})=a_{r} \exp \left(\phi x_{r}\right)+b_{r}$ for all $r \in[m]$ for some constant $a_{r}, b_{r}, \phi \in \mathbb{R}$. As $\mathcal{C}$ is closed under composition, this implies that all functions $\mathbf{c} \in \mathcal{C}$ have this property, and we retrieve case 2 of Lemma 12 . 


\section{The Complexity of Pure Nash Equilibria in Resource Graph Games}

In this section, we discuss the computational complexity of decision problems that arise in the context of pure Nash equilibria in resource graph games. First, we consider games that satisfy the requirements of Theorem 1 and, thus, always have a pure Nash equilibrium. We show that even though a pure Nash equilibrium always exists, it is coNP-complete to decide for a given game and a given strategy profile $x$ whether $x$ is a pure Nash equilibrium. This holds even for games with a single player, in which the strategy set of the player corresponds to the edge set of the sets of path between two designated vertices $s$ in $t$ in a graph where the edges are associated with the resources, and in games where the cost of each resource depends only on the load of one (other) resource. We note that this hardness result is in contrast to the subclasses of action graph games or local effect games. For these games, every strategy of every player is a singleton. This yields a simple polynomial algorithm deciding whether a given strategy profile $x$ is a pure Nash equilibrium. The algorithm computes for every player $i$ and every alternative strategy $\mathbf{y}_{i}$ the private $\operatorname{cost} \pi_{i}\left(\mathbf{y}_{i}, x_{-i}\right)$. If for one $\pi_{i}\left(\mathbf{y}_{i}, x_{-i}\right)<\pi_{i}(x)$, then $x$ is not a pure Nash equilibrium. If no such profitable deviation is found, then $x$ is a pure Nash equilibrium. For resource graph games, this algorithm is finite, but not necessarily polynomial, since the set of strategies of each player may be exponential in the size of the instance.

Theorem 3. It is coNP-complete to determine whether a given strategy profile of a resource graph game $G$ is a pure Nash equilibrium. This holds even when $G$ satisfies any number of the following properties:

1. G has only a single player;

2. the cost function c fulfills the requirements of Theorem 1;

3. $X_{1}=\left\{\mathbf{1}_{S}: S\right.$ is an $s$-t-path $\}$ for a given directed graph with edge set $R$;

4. $\left|B_{r}\right|=1$ for all $r \in R$.

Proof. Membership in the class coNP is clear since when $x$ is not a pure Nash equilibrium, then there is a player $i$ and an alternative strategy $\mathbf{y}_{i} \in X_{i}$ such that $\pi_{i}\left(\mathbf{y}_{i}, x_{-i}\right)<\pi_{i}(x)$. This inequality can be checked in polynomial time, thus, $\mathbf{y}_{i}$ is a polynomial certificate for $x$ not being a pure Nash equilibrium.

To show that the problem is coNP-hard, we reduce from ForBIDDEN-PAIRS- $s$ - $t$-PATH, which is known to be NP-hard (Gabow, Maheshwari, \& Osterweil, 1976). Given a digraph $G=(V, E)$, two nodes $s, t \in V$, and a collection of edge pairs $\left\{e_{1}, e_{1}^{\prime}\right\}, \ldots,\left\{e_{k}, e_{k}^{\prime}\right\}$, it is NP-hard to decide whether there exists an $s$-t-path $P$ such that $\left|P \cap\left\{e_{i}, e_{i}^{\prime}\right\}\right| \leq 1$ for all $i \in[k]$. Given an instance of ForbidDen-PAIRS- $s$ - $t$-PATHs, we construct a resource graph game as follows. We add an additional edge $e_{0}$ from $s$ to $t$ with cost $c_{e_{0}}(\mathbf{x})=x_{e_{0}}$ for all $\mathbf{x} \in \mathbb{R}^{E}$, and let $R=E \cup\left\{e_{0}\right\}$. For every edge $e \in E$ and $\mathbf{x} \in \mathbb{R}^{R}$, we define

$$
c_{e}(\mathbf{x})= \begin{cases}x_{e_{i}^{\prime}} & \text { if } e=e_{i} \text { for some } i \in[k], \\ x_{e_{i}} & \text { if } e=e_{i}^{\prime} \text { for some } i \in[k], \\ 0 & \text { otherwise. }\end{cases}
$$


The game has a single player whose strategy space corresponds to the set of $s$-t-paths in $G$. It is easy to see that player 1 has a strategy of cost 0 if and only if there exists a path avoiding all forbidden pairs, and that all other strategies cost at least 1 . As a consequence, the strategy $\mathbf{x}_{1}=\mathbf{1}_{e_{0}}$ where player 1 uses edge $e_{0}$ only is a pure Nash equilibrium if and only if there is no path avoiding all forbidden pairs, showing the claimed result.

We proceed to show that in games that do not satisfy the requirements of Theorem 1, the decision whether a pure Nash equilibrium exists is $\Sigma_{2}^{\mathrm{p}}$-complete. This implies that this problem lies at the second level of the polynomial hierarchy and, thus, cannot be contained in NP, unless the polynomial hierarchy collapses. The upshot of this result is that this decision problem is considerably harder than NP-complete problems.

Theorem 4. It is $\Sigma_{2}^{\mathrm{p}}$-complete to determine whether a given resource graph game $G$ has a pure Nash equilibrium. This holds even when $G$ satisfies any number of the following properties:

\section{G has only two players;}

2. $\mathbf{c}$ is affine linear, i.e., $\mathbf{c}(\mathbf{x})=\mathbf{A} \mathbf{x}+\mathbf{b}$ with $\mathbf{A} \in \mathbb{R}^{|R| \times|R|}, b \in \mathbb{R}_{\geq 0}^{|R|}$;

3. $X_{i}=\left\{\mathbf{1}_{S}: S\right.$ is an $s^{i}-t^{i}$-path $\}$ for every player $i$ for a given directed graph with edge set $R$.

Proof. The class $\Sigma_{2}^{\mathrm{p}}$ contains the problems of the form where an existential quantification is followed by a universal quantification over a polynomially bounded and polynomially decidable relation. The existence of a pure Nash equilibrium can be phrased exactly like this since it asks for a strategy profile $x$ such that for all strategy profiles $y=\left(\mathbf{y}_{i}, x_{-i}\right)$ for some $i \in N$ and $\mathbf{y}_{i} \in X_{i}$, we have $\pi_{i}(y) \geq \pi_{i}(x)$. The latter condition can be checked in polynomial time thus putting the problem to decide the existence of a pure Nash equilibrium in $\Sigma_{2}^{\mathrm{p}}$.

To show that the problem is also complete for this class, we reduce from 2-QUANTIFIED 3-DNF-SATisfiabiLity. In the problem, we are given two disjoint sets $Y^{1}=\left\{y_{1}^{1}, \ldots, y_{n}^{1}\right\}$ and $Y^{2}=\left\{y_{1}^{2}, \ldots, y_{n}^{2}\right\}$ of Boolean variables, and a Boolean formula $\phi$ over $Y^{1} \cup Y^{2}$ in disjunctive normal form where each of the conjunctive clauses consists of exactly three distinct literals. The computational problem to decide whether there is an assignment of the variables $y_{1}^{1}, \ldots, y_{n}^{1}$ such that for all assignments of the variables $y_{1}^{2}, \ldots, y_{n}^{2}$ the formula $\phi\left(y_{1}^{1}, \ldots, y_{n}^{1}, y_{1}^{2}, \ldots, y_{n}^{2}\right)$ is true is $\Sigma_{2}^{\mathrm{p}}$-complete as shown by Stockmeyer (1977).

Instead of working with the Boolean formula in disjunctive normal form, we work with the negation $\psi\left(y_{1}^{1}, \ldots, y_{n}^{1}, y_{1}^{2}, \ldots, y_{n}^{2}\right)=\neg \phi\left(y_{1}^{1}, \ldots, y_{n}^{1}, y_{1}^{2}, \ldots, y_{n}^{2}\right)$. From the disjunctive normal form of $\phi$, a conjunctive normal form of $\psi$ can be obtained in linear time.

We proceed to construct a two-player game that has a pure Nash equilibrium if and only if a given instance of 2-QUANTIFIED 3-DNF-SATISFIABILITY has a solution, thus proving the theorem. In the construction, the resources will correspond to edges of the graph depicted in Figure 6.

We first describe the resources and strategies of the first player. That player will essentially control the variables $y_{1}^{1}, \ldots, y_{n}^{1}$ of the Boolean formula. 

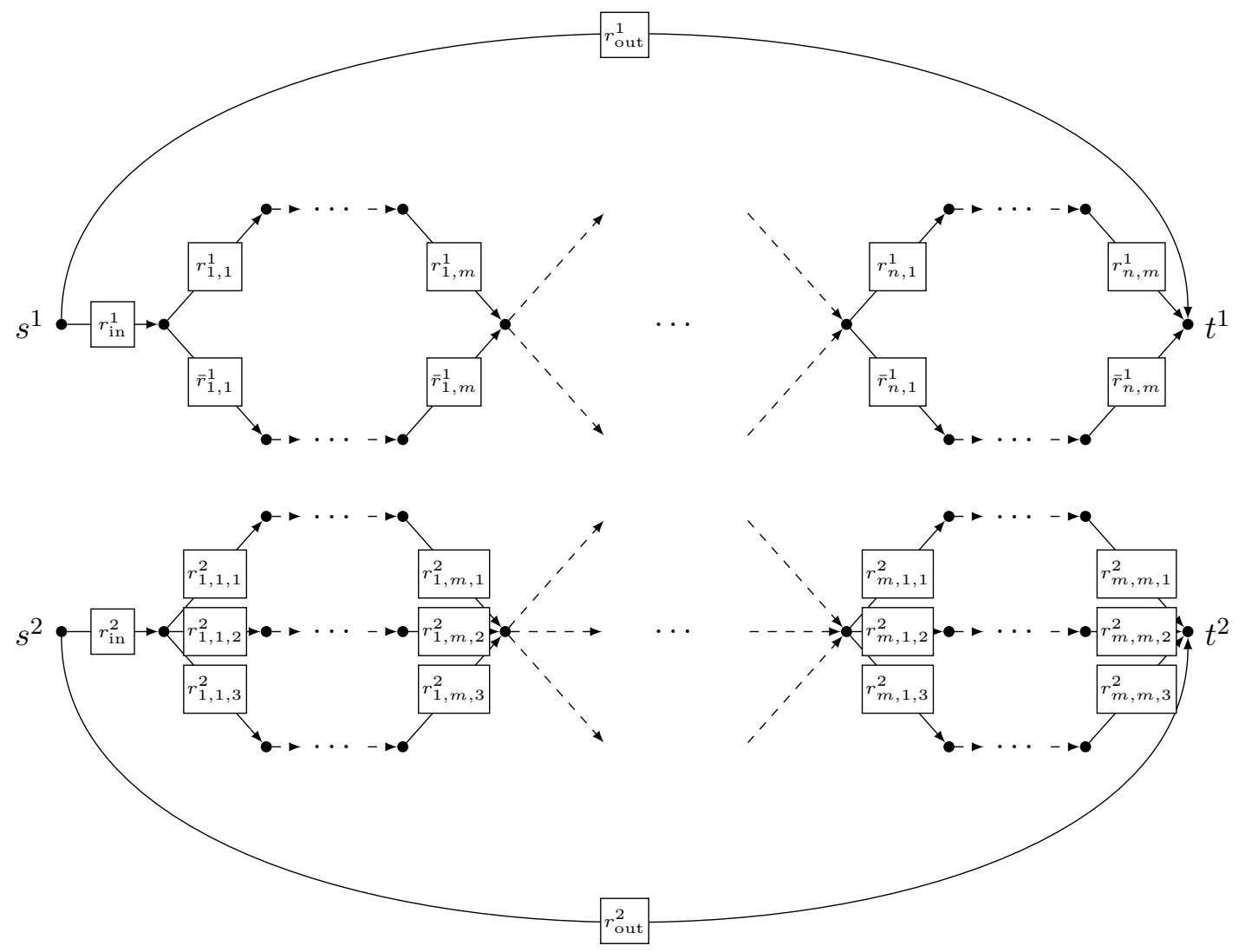

Figure 6: Construction for the proof of $\Sigma_{2}^{\mathrm{p}}$-hardness.

For each variable $y_{i}^{1}, i \in[n]$ and clause $j \in[m]$ we introduce two resources $r_{i, j}^{1}$ and $\bar{r}_{i, j}^{1}$. Resources $r_{i, j}^{1}$ model the setting of variable $y_{i}^{1}$ to true and resources $\bar{r}_{i, j}^{1}$ model the setting of variable $y_{i}^{1}$ to false. Furthermore, we introduce two additional resources $r_{\text {in }}^{1}$ and $r_{\text {out }}^{1}$. The strategy set of player 1 corresponds to the edge sets of all $s^{1}-t^{1}$-paths of the graph in Figure 6. Every $s^{1}-t^{1}$-path contains either the in-resource $r_{\text {in }}^{1}$ or the out-resource $r_{\text {out }}^{1}$. The path containing the out-resource does not contain any further resources. All paths containing the in-resource correspond to a truth assignment of the variables $Y^{1}$ in the following way. Each such path for every variable $y_{i}^{1}, i \in[n]$ contains either the resources $r_{i, j}^{1}$ for all $j \in[m]$ (interpreted as setting $y_{i}^{1}=$ true) or contains the resources $\bar{r}_{i, j}^{1}$ for all $j \in[m]$ (interpreted as setting $y_{i}^{1}=$ false).

We proceed to explain the strategy of player 2 and the resources contained in it. Let $\left(z_{j, 1} \vee z_{j, 2} \vee z_{j, 3}\right)$ with $j \in[m]$ be a clause of $\psi$. It is without loss of generality to assume that no variable appears multiple times in the literals for a clause. We introduce $3 m$ literal resources $r_{j, 1,1}^{2}, \ldots, r_{j, m, 1}^{2}, r_{j, 1,2}^{2}, \ldots, r_{j, m, 2}^{2}$, and $r_{j, 1,3}^{2}, \ldots, r_{j, m, 3}^{2}$. Furthermore, also player 2 has an in-resource $r_{\text {in }}^{2}$ and an out-resource $r_{\text {out }}^{2}$. The strategy set of player 2 corresponds to 
the edge sets of all $s^{2}-t^{2}$-paths of the graph in Figure 6. Every $s^{2}-t^{2}$-path contains either the in-resource $r_{\text {in }}^{2}$ or the out-resource $r_{\text {out }}^{2}$. The path containing the out-resource does not contain any further resources. Every path containing the in-resource contains for every clause $j \in[m]$ one set of literal resources $r_{j, j^{\prime}, k}^{2}$ for all $j^{\prime} \in[m]$ and some $k \in[3]$.

The variable resources $r_{i, j}^{1}$ and $\bar{r}_{i, j}^{1}$ have $\operatorname{cost} c_{r_{i, j}^{1}}(\mathbf{x})=c_{\bar{r}_{i, j}^{1}}(\mathbf{x})=0$ for all $i \in[n], j \in[m]$, and $\mathbf{x} \in \mathbb{N}^{R}$.

We next define the costs for the literal resources. The cost functions on the literal resources depend on whether the corresponding literal belongs to a variable in $Y^{1}$ or $Y^{2}$. We set

$$
c_{r_{j, j^{\prime}, k}^{2}}(\mathbf{x})= \begin{cases}x_{r_{i, j}^{1}} & \text { if } z_{j, k}=\neg y_{i}^{1} \text { for some } i \in[n], \\ x_{\bar{r}_{i, j}^{1}} & \text { if } z_{j, k}=y_{i}^{1} \text { for some } i \in[n], \\ \sum_{k^{\prime} \in[3]: z_{j, k}=\neg z_{j^{\prime}, k^{\prime}}} x_{r_{j^{\prime}, j, k^{\prime}}^{2}} & \text { otherwise }\end{cases}
$$

for all $j, j^{\prime} \in[m], k \in[3]$, and $\mathbf{x} \in \mathbb{N}^{R}$. The intuition behind these cost functions is that the cost of the literal edges $r_{j, 1, k}^{2}, \ldots, r_{j, m, k}^{2}$ corresponding to literal $z_{j, k}$ are increased if either the corresponding variable is controlled by player 1 and player 1 chooses the other variable assignment for that variable, or the variable is controlled by player 2 and the player chooses a conflicting literal edge for another clause. Further note that in the latter case, for every resource $r_{j, j^{\prime}, k}^{2}$ with $z_{j, k}$ not corresponding to a variable in $Y^{1}$, there is at most one $k^{\prime} \in[3]$ such that $z_{j, k}=\neg z_{j^{\prime}, k^{\prime}}$ since every variable appears at most once per clause. Finally, we set $c_{r_{\text {out }}^{2}}(\mathbf{x})=1, c_{r_{\text {in }}^{2}}(\mathbf{x})=2 x_{r_{\text {out }}^{1}}, c_{r_{\text {in }}^{1}}(\mathbf{x})=2 x_{r_{\text {in }}^{2}}$, and $c_{r_{\text {out }}^{1}}(\mathbf{x})=1 / 2$ for all $\mathbf{x} \in \mathbb{N}^{R}$. Note that $\left|B_{r}\right| \leq 1$ for all $r \in R$.

We claim that the thus defined game has a pure Nash equilibrium if and only if there is an assignment of the variables $y_{1}^{1}, \ldots, y_{n}^{1}$ such that for all assignments of the variables $y_{1}^{2}, \ldots, y_{n}^{2}$, we have that $\psi\left(y_{1}^{1}, \ldots, y_{n}^{1}, y_{1}^{2}, \ldots, y_{n}^{2}\right)$ is false.

Let us first assume that such an assignment exists. Let $T \subseteq Y^{1}$ be the subset of variables of player 1 that are set to true in such an assignment. Let

$$
\mathbf{x}_{1}=\mathbf{1}_{r_{\mathrm{in}}^{1}}+\sum_{i \in T} \mathbf{1}_{r_{i}^{1}}+\sum_{i \in[n] \backslash T} \mathbf{1}_{\bar{r}_{i}^{1}}
$$

be the corresponding strategy. Further, consider the strategy of player 2 that uses the out-resource, i.e.,

$$
\mathbf{x}_{2}=\mathbf{1}_{r_{\text {out }}^{2}} .
$$

We proceed to show that $\left(\mathbf{x}_{1}, \mathbf{x}_{2}\right)$ is a pure Nash equilibrium. Player 1 obviously has no profitable deviation from $\left(\mathbf{x}_{1}, \mathbf{x}_{2}\right)$ as her private cost is 0 and all costs are non-negative. For player 2, we have $\pi_{2}\left(\mathbf{x}_{1}, \mathbf{x}_{2}\right)=1$, so if there is a strategy $\mathbf{y}_{2} \in X_{2}$ with $\pi_{2}\left(\mathbf{x}_{1}, \mathbf{y}_{2}\right)<1$, then it must be the case that player 2 uses $r_{\text {in }}^{2}$ in $\mathbf{y}_{2}$. This, implies that for each clause $j \in[m]$, player 2 uses one of the sets of literal resources $\left\{r_{j, 1, k_{j}}, \ldots, r_{j, m, k_{j}}\right\}$ for some $k_{j} \in[3]$. Since there is no satisfying assignment, player 2 must then use either a set of literal resources $\left\{r_{j, 1, k}, \ldots, r_{j, m, k}\right\}$ associated with a variable of player 1 that player 1 did not set accordingly (incurring a cost of $m$ ), or player 2 must have conflicting assignments for a variable in $Y^{2}$ 
(incurring a cost of 2). In either case, $\mathbf{y}_{2}$ is not a profitable deviation for player 2 and, hence, $\left(\mathbf{x}_{1}, \mathbf{x}_{2}\right)$ is a pure Nash equilibrium.

Next, let us assume that such an assignment does not exist, i.e., for all assignments of the variables in $Y^{1}$, there is an assignment of the variables in $Y^{2}$ that satisfies $\psi$. We proceed to show that in this case, the game does not have a pure Nash equilibrium. For a contradiction, let us assume that $\left(\mathbf{x}_{1}, \mathbf{x}_{2}\right)$ is a pure Nash equilibrium and let us first consider the case that player 1 uses resource $r_{\text {in }}^{1}$ in $\mathbf{x}_{1}$. For every variable $i \in[n]$, player 1 chooses either all resources $r_{i, j}^{1}$ for all $j$ or all resources $\bar{r}_{i, j}^{1}$ for all $j$ and the strategy of player 1 can be interpreted as an assignment of the variables in $Y^{1}$. For every such assignment, there is an assignment of the variables in $Y^{2}$ such that $\psi$ is satisfied, i.e., for each clause $j \in[m]$ there is a literal $z_{j, k_{j}}$ with $k_{j} \in[3]$ satisfying $\psi$. Consider the corresponding strategy

$$
\mathbf{x}_{2}=\mathbf{1}_{r_{\mathrm{in}}^{2}}+\sum_{j \in[m]} \sum_{j^{\prime} \in[m]} \mathbf{1}_{r_{j, j^{\prime}, k_{j}}}
$$

of player 2. By construction, $\pi_{2}\left(\mathbf{x}_{1}, \mathbf{x}_{2}\right)=0$. Since resource $r_{\text {out }}^{2}$ incurs a cost of 1 for player 2, strategies $\mathbf{x}_{2}$ with $x_{2, r_{\text {in }}^{2}}=1$ are the only candidates for a pure Nash equilibrium $\left(\mathbf{x}_{1}, \mathbf{x}_{2}\right)$. However, since $x_{2, r_{\mathrm{in}}^{2}}=1$, player 1 has a cost of 2 on resource $r_{\text {in }}^{1}$ making it profitable to switch to $r_{\text {out }}^{1}$ while keeping the rest of the strategy. This shows that there is no pure Nash equilibrium with $x_{1, r_{\text {in }}^{1}}=1$.

Next, consider the case that $x_{1, r_{\text {out }}^{1}}=1$. In that case, we have that any strategy of player 2 using $r_{\text {in }}^{2}$ has a cost of at least 2 while the strategy $\mathbf{x}_{2}=\mathbf{1}_{r_{\text {out }}^{2}}$ has a cost of 1 . We conclude that player 2 is not using $r_{\text {in }}^{2}$. In that case, however, player 1 could switch from $r_{\text {out }}^{1}$ to any of the paths containing $r_{\text {in }}^{1}$, obtaining a cost of 0 . We conclude that also in this case, a Nash equilibrium does not exist.

\section{Conclusion}

In this paper, we gave a complete characterization of a set of consistent cost maps $\mathcal{C}$ having the property that any resource graph game with cost maps contained in $\mathcal{C}$ admits pure Nash equilibria. We complemented our characterizations by several hardness results regarding the equilibrium existence problem in terms of recognizing a strategy profile as pure Nash equilibrium or to decide their existence. Our characterizations require that $\mathcal{C}$ is closed under composition, that is, for any two cost maps $\mathbf{c}, \mathbf{c}^{\prime} \in \mathcal{C}$ we have $\mathbf{c} \oplus \mathbf{c}^{\prime} \in \mathcal{C}$. While quite natural, this assumption allows to construct examples used for proving the only if direction of our characterization. It is an interesting open question to give a complete characterization of consistency of cost functions without this assumption. Another interesting research direction is to consider restricted strategy spaces such as matroids or other graph structures and derive alternative characterizations of consistency of cost functions.

\section{Acknowledgments}

We thank the anonymous referees for their comments that helped to improve the presentation of the paper. This work was supported by Deutsche Forschungsgemeinschaft 
(DFG - German Research Foundation) under grants HA 8041/4-1, MA 8439/1-1, and under Germany's Excellence Strategy - The Berlin Mathematics Research Center MATH+ (EXC-2046/1, project ID: 390685689).

\section{References}

Ackermann, H., Röglin, H., \& Vöcking, B. (2008). On the impact of combinatorial structure on congestion games. J. ACM, 55(6), 1-22.

Ackermann, H., Röglin, H., \& Vöcking, B. (2009). Pure Nash equilibria in player-specific and weighted congestion games. Theoret. Comput. Sci., 410(17), 1552-1563.

Bhat, N. A. R., \& Leyton-Brown, K. (2004). Computing Nash equilibria of action-graph games. In Chickering, D. M., \& Halpern, J. Y. (Eds.), Proc. 20th Conf. on Uncertainty in Artif. Intell. (UAI), pp. 35-42.

Blum, B., Shelton, C., \& Koller, D. (2006). A continuation method for Nash equilibria in structured games. J. Artificial Intelligence Res., 25, 457-502.

Chan, H., \& Jiang, A. X. (2018). An FPTAS for computing Nash equilibrium in resource graph games. In Lang, J. (Ed.), Proc. 27th Internat. Joint Conf. Artif. Intell. (IJCAI), pp. $152-158$.

Dafermos, S. (1971). An extended traffic assignment model with applications to two-way traffic. Transportation Sci., 5, 366-389.

Dafermos, S. (1972). The traffic assignment problem for multiclass-user transportation networks. Transportation Sci., 6(1), 73-87.

Daskalakis, C., Schoenebeck, G., Valiant, G., \& Valiant, P. (2009). On the complexity of Nash equilibria of action-graph games. In Proc. 20th Annual ACM-SIAM Sympos. on Discrete Algorithms (SODA), pp. 710-719.

Dunkel, J., \& Schulz, A. S. (2008). On the complexity of pure-strategy Nash equilibria in congestion and local-effect games. Math. Oper. Res., 33(4), 851-868.

Fotakis, D., Kontogiannis, S., \& Spirakis, P. (2005). Selfish unsplittable flows. Theoret. Comput. Sci., 348(2-3), 226-239.

Gabow, H. N., Maheshwari, S. N., \& Osterweil, L. J. (1976). On two problems in the generation of program test paths. IEEE Trans. Softw. Eng., SE-2(3), 227-231.

Goemans, M. X., Mirrokni, V., \& Vetta, A. (2005). Sink equilibria and convergence. In Proc. 46th Annual IEEE Sympos. Foundations Comput. Sci. (FOCS), pp. 142-154.

Gottlob, G., Greco, G., \& Scarcello, F. (2005). Pure Nash equilibria: Hard and easy games. J. Artificial Intelligence Res., 24, 357-406.

Harks, T., \& Klimm, M. (2012). On the existence of pure Nash equilibria in weighted congestion games. Math. Oper. Res., 37(3), 419-436.

Harks, T., Klimm, M., \& Möhring, R. (2011). Characterizing the existence of potential functions in weighted congestion games. Theory Comput. Syst., 49(1), 46-70. 
Jiang, A. X., Chan, H., \& Leyton-Brown, K. (2017). Resource graph games: A compact representation for games with structured strategy spaces. In Singh, S. P., \& Markovitch, S. (Eds.), Proc. 31st Conf. on Artif. Intell. (AAAI), pp. 572-578.

Jiang, A. X., \& Leyton-Brown, K. (2007). Computing pure Nash equilibria in symmetric action graph games. In Proc. 22nd Conf. on Artif. Intell. (AAAI), pp. 79-85.

Jiang, A. X., Leyton-Brown, K., \& Bhat, N. A. R. (2011). Action-graph games. Games Econom. Behav., 71(1), 141-173.

Kearns, M., Littman, M., \& Singh, S. (2001). Graphical models for game theory. In Proc. 17th Conf. on Uncertainty in Artif. Intell. (UAI), pp. 253-260.

Kuhn, H. W. (1953). Extensive games and the problem of information. In Kuhn, H. W., \& Tucker, A. W. (Eds.), Contributions to the theory of games, Vol. II, Vol. 28 of Annals of Mathematics Studies, pp. 193-216. Princeton University Press.

Leyton-Brown, K., \& Tennenholtz, M. (2003). Local-effect games. In Gottlob, G., \& Walsh, T. (Eds.), Proc. 18th Internat. Joint Conf. Artif. Intell. (IJCAI), pp. 772-780.

Libman, L., \& Orda, A. (2001). Atomic resource sharing in noncooperative networks. Telecommun. Syst., 17(4), 385-409.

Milchtaich, I. (1996). Congestion games with player-specific payoff functions. Games Econom. Behav., 13(1), 111-124.

Monderer, D., \& Shapley, L. S. (1996a). Fictitious play property for games with identical interests. J. Econom. Theory, 68(1), 258-265.

Monderer, D., \& Shapley, L. S. (1996b). Potential games. Games Econom. Behav., 14(1), $124-143$.

Nash, J. (1950). Equilibrium points in n-person games. Proc. Natl. Acad. Sci. USA, 36, $48-49$.

Ortiz, L. E. (2020). On sparse discretization for graphical games. J. Artificial Intelligence Res., 69, 67-84.

Panagopoulou, P., \& Spirakis, P. (2006). Algorithms for pure Nash equilibria in weighted congestion games. ACM J. Exp. Algorithmics, 11, 1-19.

Perakis, G. (2007). The "price of anarchy" under nonlinear and asymmetric costs. Math. Oper. Res., 32(3), 614-628.

Rosenthal, R. (1973). A class of games possessing pure-strategy Nash equilibria. Internat. J. Game Theory, 2(1), 65-67.

Smith, M. J. (1979). The existence, uniqueness and stability of traffic equilibria. Transp. Res. Part B, 13(4), 295-304.

Stockmeyer, L. J. (1977). The polynomial-time hierarchy. Theoret. Comput. Sci., 3, 1-22.

Thompson, D. R. M., \& Leyton-Brown, K. (2017). Computational analysis of perfectinformation position auctions. Games Econom. Behav., 102, 583-623. 